\title{
Optimal Voting Schemes with Costly Information Acquisition*
}

\author{
Alex Gershkov ${ }^{\dagger}$ \\ Department of Economics, Hebrew University of Jerusalem
}

Balázs Szentes ${ }^{\ddagger}$

Department of Economics, University of Chicago

\begin{abstract}
This paper analyzes a voting model where (i) there is no conflict of interest among the voters, and (ii) information acquisition is costly and unobservable. The optimal mechanism is shown to be sequential. The social planner asks, at random, one voter at a time to invest in information and to report the resulting signal. Voters are informed of neither their position in the sequence nor the reports of previous voters. Obeying the planner by investing and reporting truthfully is optimal for voters. The ex-ante optimal voting scheme among the ex-post efficient ones is characterized. In this scheme, the social planner stops aggregating information and makes a decision when the precision of his posterior exceeds a cut-off which decreases with each additional report. It is also shown that if the cost of information acquisition is small, then the ex-ante optimal mechanism is sometimes necessarily ex-post inefficient.
\end{abstract}

\section{Introduction}

In many environments, groups rather than individuals make decisions. Information about the desirability of the possible decisions is often dispersed: Individual group members must separately

\footnotetext{
${ }^{*}$ We are grateful for helpful discussions to Eddie Dekel, Jeff Ely, Tim Federsen, Ali Hortacsu, Motty Perry, Phil Reny, Rob Shimer, and Hugo Sonnenschein. We thank seminar participants at the University of Chicago, Iowa State University, Tel Aviv University, Hebrew University, Boston University, Northwestern, and Queen's University for comments.

$\dagger$ Mount Scopus, Jerusalem, 91905, Israel. Email: mar@pob.huji.ac.il.

$\ddagger 1126$ E. 59th Street, Chicago, IL 60657, USA. Email: szentes@uchicago.edu.
} 
acquire information about the alternatives. The group is more likely to make the right decision if it aggregates individuals' information. However, it often is costly for individuals to acquire information, and whether they have done so is often unobservable. Hence, even when there is no conflict of interest among the group members, an individual may not want to acquire information if the probability that she will influence the final decision is too small compared with her cost. That is, she compares the cost of information only to her own benefit, not to society's. The naturally arising mechanism design problem is to design an information aggregation scheme that maximizes the group's expected gain from making the right decision, net of the total expected cost of acquiring information. The scheme must make the probability that an individual is pivotal sufficiently large, compared with the cost of information, that individuals have adequate incentive to collect information. This paper analyzes optimal voting schemes in such environments.

Small groups make decisions in many economic and political situations. Consider, for instance, a recruiting committee in an academic department. The committee's objective is to identify the candidate who best fits a vacant job. (For example, the committee may want to hire somebody who can teach and research a narrowly defined field.) The committee members review applicants' CVs, teaching evaluations, and research papers. The opportunity cost of this work may be substantial because time spent reading applications cannot be devoted to a member's own research. The chair of such a committee faces the following dilemma: On the one hand, to make an accurate assessment, he wants several committee members to review the same candidate. On the other hand, if several members review the same candidate, the members may lack the incentive to work hard. Each member understands that, even if she does not spend much time reviewing the candidate and hence forms an inaccurate opinion, the others' reports may induce a correct final decision. Thus the members will not exert effort if their reports are not likely to be decisive.

Consider, also, the board of a company that must evaluate a potential merger. The board members' goal should be to maximize the value of their company. Properly forecasting the consequences of a merger requires detailed analysis of data about the target firm, such as its past performance, the value of its capital stock, its market share, and so on. The board may hire experts to help with this analysis, but the board members ultimately must reach their own conclusions. If a board member trusts other members' assessments, she has no incentive to invest energy in forming her own opinion; she will prefer to let the other members decide. But if all members think this way, the final decision may be very poor.

Another example is a legislative committee considering whether to build a highway. The committee must analyze the proposed road's costs and benefits, which are complex. A new highway may reduce not only the time residents spend traveling but also the cost of local trade, which in turn can reduce the prices of some commodities. A new highway may also lead to more tourism. On the other hand, the government must levy taxes to finance the project. New taxes have both political and economic consequences. In addition, a new highway may generate heavier traffic, which may increase air and soil pollution. Even understanding the complicated calculation of the 
project's net benefit requires the committee members to incur significant costs. If they do not live near the site of the proposed highway, they may simply leave the decision to other committee members.

Finally, suppose that a patient is diagnosed with lung cancer. Two treatments are available: surgery and chemotherapy. The probability of each treatment's success depends on the type and size of the tumor, the patient's other medical problems, her age, etc. A group of doctors, each an expert on some of these factors, must agree on the best treatment to recommend to the patient. But, as in the previous examples, making a responsible recommendation is costly; each doctor may want to save that cost if she trusts her colleagues' judgment.

In all of these cases, even though the committee members have the same objective, decisions may be inefficient because members may prefer to rely on others' opinions instead of acquiring information themselves. We view this problem as a social choice theoretical one. Most of social choice theory, however, focuses on efficient decision making in the presence of conflicts of interest among the members of a society. If individuals disagree about the merits of possible social alternatives, they may want to misrepresent their preferences to influence the final decision, which can lead to inefficient outcomes. We believe that another source of inefficiency is the difficulty of aggregating information. Hence, in this paper, we completely abstract from potential conflicts of interest and focus solely on the costly and unobservable nature of information acquisition. Surprisingly, the modern Bayesian mechanism design literature has not yet fully explored this important and basic line of research.

We set up probably the simplest possible model where (i) there is no conflict of interest among voters, and (ii) information acquisition is costly and unobservable. A theoretical interest of our model is that the Revelation Principle cannot be applied. The voters have no information, and hence no type, to start with. Therefore, the optimal mechanism cannot be based simply on asking the voters to report their information. The total amount of information aggregated in the optimal mechanism is an endogenous object of our interest.

We show that for any equilibrium in any voting scheme, there exists another outcome-equivalent mechanism and equilibrium with the following properties. First, the voters are randomly ordered. Next, the voters are asked sequentially to acquire and report information. A voter receives no information about either her position in the sequence or the actions of previous voters. After each voter's report, either a final decision is made or an additional voter is asked. A voter's set of actions is the set of signals she can possibly draw and report about the state of the world. (We call such a mechanism a canonical mechanism.) In equilibrium, whenever a voter is asked to acquire information, she does so and reports the information truthfully.

Most of this paper focuses on ex-post efficient mechanisms. Committing to ex-post inefficient decisions is often impossible or absurd in the environments of our interest. (It is unlikely that a group of doctors can commit to recommending a treatment that will almost surely kill the patient; a rejected job candidate may sue the academic department if she can prove that she is 
better qualified than the successful applicant.) Therefore, we characterize optimal voting schemes among the ex-post efficient ones. The optimal scheme in this class has a particularly simple form. Essentially it can be characterized by a cut-off function, $f$, mapping the number of solicited voters to the precision of the posterior distribution over states of the world. If, after receiving reports from $n$ voters, the posterior has precision above the cut-off $f(n)$, information acquisition stops and a decision is made. Otherwise, an additional voter is asked to acquire information and to report her resulting signal. Our main result is that $f$ is nonincreasing. Consequently, with each additional report the cut-off precision of the posterior decreases. That is, less precise information is required to induce a decision as the number of solicited voters increases We also show that, if the cost of information acquisition is small, then, surprisingly, the ex-ante optimal mechanism is often ex-post inefficient. That is, ex-post inefficient decisions can be used as a threat to induce voters to acquire information.

We view this mechanism design problem as a particularly important and applied one. This line of research may not only deepen understanding of voting theory and existing information aggregation procedures but also lead to specific recommendations for improving voting schemes now in use.

Most of the existing literature has focused on voting models with exogenously given information structure. See Austen-Smith and Banks (1996), Chwe (1999), Federsen and Pesendorfer (1996, 1997, 1998), Li, Rosen, and Suen (2001), and McLennan (1998). For example, Dekel and Piccione (2000) compare sequential and simultaneous voting and find equivalence between different voting schemes in terms of equilibrium outcome. In particular, they find that for any choice rule, there is a Pareto best equilibrium outcome that is the same whether voting is sequential or simultaneous. In contrast, this paper shows that sequential schemes always dominate simultaneous ones when information acquisition is costly.

There is a growing literature on information acquisition in mechanism design. Most of this literature deals with auction and public good models where utilities are transferable, unlike in voting models. See Bergemann and Valimaki (2002), Persico (2000), Milgrom (1981), and the references therein. These papers focus on simultaneous information acquisition. Their goal is to analyze the incentives to acquire information in different classical mechanisms.

Persico (2004) considers two-stage voting games of the following form. At the first stage, all committee members simultaneously decide whether to acquire a noisy signal about the state of the world or to remain uninformed. At the second stage, they vote. The author analyzes the optimal voting scheme among threshold voting rules and the optimal size of the committee. Gerardi and Yariv (2003) analyze environments similar to the ones in Persico (2004). They enrich the model by introducing a communication stage before voting. This cheap talk stage can make the mechanism more efficient. Mukhopadhaya (2003) analyzes the effect of committee size on the accuracy of the final decision. He shows that in symmetric mixed-strategy equilibria, increasing the committee size may lead to a less accurate decision. In asymmetric pure-strategy equilibria, however, changing 
the committee size does not affect the accuracy of the final decision.

All of the papers described in the previous paragraph analyze settings with homogeneous preferences. Cai (2003) allows for heterogeneous preferences with both non-verifiable information acquisition and costly participation in a committee. In this model, members are supposed to report their information to a principal. The principal makes the final decision based on the reports. Cai (2003) characterizes the optimal committee size when the signals as well as the decision are continuous variables and the principal uses the mean decision rule to determine the final decision. Li (2001) considers a committee with a fixed number of members. Each member acquires a noisy signal. The signals become publicly observable by all the other committee members after acquisition. He analyzes the properties of the optimal decision rule for the case of simultaneous information acquisition. To provide incentives for acquiring information, it is optimal to distort the decision rule away from the ex-post optimal one. Cai (2003) treats the decision rule as given and focuses on the committee size; Li (2001) does just the opposite. Austen-Smith and Federsen (2002) also consider a model where the voters have heterogeneous preferences over two alternatives. Their model allows the voters to communicate prior to voting. They show that unanimous voting makes it impossible to reveal all the private information.

Smorodinsky and Tennenholtz (2003) analyze the problem of free riding in multi-party computations. A group of people has to compute the value of a certain function. Each person must incur a cost to retrieve a piece of input. The agents face a free-rider problem similar to ours: If the probability that a single piece of input affects the value of the function is too small, an individual prefers not to incur the cost and provides an input at random. Smorodinsky and Tennenholtz (2003) analyze mechanisms in which the true value of the function is computed with probability one. Therefore, unlike in our paper, there is no trade-off between the total cost of information acquisition and the accuracy of the final decision. On the other hand, Smorodinsky and Tennenholtz (2003) derive a canonical mechanism, independently from us, that has similar attributes to our canonical mechanism. In particular, they show that the mechanism can be assumed to be sequential and that each player is told only whether she should or should not retrieve the input.

The paper is organized as follows. Section 2 describes the model. Section 3 characterizes the first-best voting scheme. Section 4 characterizes the canonical mechanisms, explicitly derives the incentive compatibility constraint, and proves some basic properties of the optimal mechanisms. The main results are in Section 5. Section 6 concludes. Most of the proofs are relegated to the appendixes.

\section{The Model}

There is a population consisting of $N(\in \mathbb{N})$ individuals. There are two possible states of the world: $H$ and $L$. Each of these states occurs with probability one-half. The society must take an action, 
either $a_{H}$ or $a_{L}$. An individual's utility is

$$
\begin{aligned}
u\left(a_{H} \mid H\right) & =u\left(a_{L} \mid L\right)=1 \\
u\left(a_{H} \mid L\right) & =u\left(a_{L} \mid H\right)=0 .
\end{aligned}
$$

Every individual can draw a signal at most once at a cost $c$. The value of a signal is either $\mathcal{H}$ or $\mathcal{L}$ and is distributed as follows:

$$
\begin{aligned}
p(\mathcal{H} \mid H) & =p(\mathcal{L} \mid L)=p>1 / 2 \\
p(\mathcal{H} \mid L) & =p(\mathcal{L} \mid H)=1-p=q .
\end{aligned}
$$

The signals are independently distributed conditional on the state of the world. Information acquisition is unobservable. (We also implicitly assume that information acquisition takes no time.)

There is a Social Planner (SP) who wants to maximize expected sum of the individuals' utilities, net of the expected total cost of information acquisition:

$$
N E u-c \bar{L}
$$

where $\bar{L}$ is the expected number of voters who collect information. The utilities are not transferable; that is, the SP cannot use a transfer scheme to induce the voters to acquire information.

It is useful to introduce the following notation. Let $\# i(s)$ denote the number of signals $i(\in$ $\{\mathcal{H}, \mathcal{L}\})$ in the signal sequence $s$.

Lemma 1 Let $s$ be a sequence of signals such that $\# \mathcal{H}(s)-\# \mathcal{L}(s)=d$. Then

$$
\begin{aligned}
& p(H \mid s)=\frac{p^{d}}{p^{d}+q^{d}} \\
& p(\mathcal{H} \mid s)=\frac{p^{d+1}+q^{d+1}}{p^{d}+q^{d}}
\end{aligned}
$$

Proof. See Appendix A.

The lemma above states that the posteriors about the state of the world and about the next signal after observing a sequence of signals depend only on the difference between the numbers of signals $\mathcal{H}$ and $\mathcal{L}$ observed so far. We introduce the following notation: $B(d)=p^{d} /\left(p^{d}+q^{d}\right)$, $R(d)=1-B(d), p(d)=\left(p^{d+1}+q^{d+1}\right) /\left(p^{d}+q^{d}\right)$, and $q(d)=1-p(d)$.

\section{The First-Best Mechanism}

This section characterizes the first-best voting scheme. That is, the optimal mechanism in which the voters do not behave strategically, and hence the scheme is not subject to incentive compatibility. Since information acquisition takes no time, the first-best mechanism can be assumed to be 
sequential. The SP asks the voters sequentially to draw signals and report them. After each voter, the SP has to decide whether to ask an additional voter or to take an action. Clearly, whenever the SP takes an action, it corresponds to the majority of the votes. That is, if the SP acts, the action is $a_{H}$ if and only if more signals $\mathcal{H}$ were reported than signals $\mathcal{L}$. The problem of the SP is a standard stochastic dynamic programming problem. From Lemma 1, it follows that there are two state variables: the difference between the number of signals received of each type, and the number of voters who have previously been asked. The reason why the number of voters already asked is a state variable can be easily illustrated with the following example. Suppose there are ten voters. Compare the following two cases. In case one, only one voter has been asked and has reported signal $\mathcal{H}$. In case two, nine voters have been asked; five of them reported signal $\mathcal{H}$, and four of them reported signal $\mathcal{L}$. By Lemma 1, the posteriors of the SP are identical in the two cases. However, unlike in case one, in case two the value of asking an additional voter is simply zero, since even if she reports signal $\mathcal{L}$, taking action $a_{H}$ will remain optimal. In general, the fewer voters remain to possibly ask, the smaller is the value of asking an additional voter given a certain posterior. Hence, conditional on the same posterior, the larger is the number of voters who have already been asked, the less likely the SP is to ask an additional voter. We formalize this argument below.

The following proposition characterizes the first-best mechanism.

Proposition 1 There exists a decreasing function $g: \mathbb{N}_{+} \rightarrow \mathbb{N}_{+}$such that

$$
\text { for all } n \in \mathbb{N}_{+}, g(n+1)=g(n) \text { or } g(n+1)=g(n)-1 \text {, and } g(N-1)=1 \text {. }
$$

If, after asking $n$ voters, the reported signal sequence is $s$ and $|\# \mathcal{H}(s)-\# \mathcal{L}(s)|=g(n)$, the $S P$ makes the majority decision. Otherwise, the SP asks an additional voter.

This result can be deduced from DeGroot (1970), see Theorem 2 and 3 in Chapter 12.5. For expositional purpose we present a short proof below.

Proof. Let $V(n, d)$ denote the set of sequences at which the number of voters already asked is $n$ and the absolute value of the difference between the numbers of different signals is $d$. Notice that not all of the states can be reached. First, $n$ must be smaller than $N$; second, $d$ can never be larger than $n$; and third, $n+d$ must be divisible by two. (For example, after asking three voters, the difference between the numbers of different signals clearly cannot be two.) Let $\mathcal{V}$ denote the set of feasible states. Let $W(n, d)$ denote the value function of the SP at state $V(n, d)$ given that he uses the optimal decision rule. That is, $W(n, d)$ denotes $N$ times the probability of making the right decision, net of the expected cost of information acquisition of those voters who will be asked after reaching $V(n, d)$. The value function $W$ can be defined recursively as follows:

$$
\begin{aligned}
W(N, d) & =N B(d) \\
W(n, d) & =\max \{N B(d), p(d) W(n+1, d+1)+q(d) W(n+1, d-1)-c\} \text { for all } 0 \leq n<N .
\end{aligned}
$$


Any continuation decision rule that is feasible at state $V\left(n_{1}, d\right)$ is also feasible at a state $V\left(n_{2}, d\right)$ if $n_{2}$ is smaller than $n_{1}$. Hence, $W(n, d)$ is a weakly decreasing function of $n$. Also observe that the SP stops asking voters at state $V(n, d)$ if and only if $W(n, d)=N B(d)$, that is, if

$$
N B(d) \geq p(d) W(n+1, d+1)+q(d) W(n+1, d-1)-c .
$$

Since the value function $W$ is decreasing in its first argument, the right-hand-side of the previous inequality is decreasing in $n$. Hence, given a certain $d$, the more voters have been already asked, the more likely it is that the SP makes a decision. For all $d \in \mathbb{N}_{+}$, define $f(d)$ as the smallest $n$ such that the SP stops asking voters at $V(n, d) \in \mathcal{V}$ and makes a decision. Since $W$ is weakly decreasing in its first argument, it follows that conditional on being at state $V(n, d)$, the SP asks an additional voter if and only if $n<f(d)$.

We claim that $f$ is a strictly decreasing function. Suppose, by contradiction, that there exists a $d$ such that $f(d) \leq f(d+1)$. This means that at state $V(f(d)-1, d+1)$, the SP asks an additional voter. (Since $V(f(d), d) \in \mathcal{V}, V(f(d)-1, d+1) \in \mathcal{V}$.) However, the report of the additional voter does not affect the decision of the SP, because conditional on continuing to ask voters at $V(f(d)-1, d+1)$, whenever the difference between the numbers of different signals is $d, n \geq f(d)$, and hence the SP makes a decision. That is, the SP makes the same decision as in $V(f(d)-1, d+1)$. This means asking voters at $V(f(d)-1, d+1)$ does not increase the probability of making the right decision but does increase the cost of acquiring information. Therefore it cannot be optimal, which is a contradiction.

For all $n \in \mathbb{N}_{+}$, let us define $g(n)$ as the smallest $d \in \mathbb{N}_{+}$such that the SP makes a decision at state $V(n, d) \in \mathcal{V}$. That is,

$$
g(n)=\min \{d \mid n \geq f(d)\}
$$

Since $f$ is strictly decreasing, $g$ is also decreasing and $g(n+1)=g(n)$ or $g(n)-1$. Since, at a state $V(N-1, d)$, asking an additional voter influences the majority decision only if $d=0$, $g(N-1)=1$.

Observe that $g(N-1)=1$ does not mean that, in the first-best mechanism, all the voters are potentially asked to acquire information. In general, the value of $g$ is one for a number much smaller than $N-1$.

\section{Preliminaries}

\section{Canonical Mechanisms}

A voting mechanism is an extensive-form game with imperfect information where the players are the voters. At a certain information set, either chance or a voter moves ${ }^{1}$. Since information

\footnotetext{
${ }^{1}$ The move of a chance does not necessarily mean the randomization of the nature. It may mean, for example,
} 
acquisition is unobservable, at any information set where a certain voter has to take an action, she can also acquire information if she has not done so at a previous information set. Furthermore, along each path of the game there must be an information set where either action $a_{H}$ or action $a_{L}$ is taken. A mechanism is said to be sequential if the voters have to take actions one after the other according to random order. Let $G$ be such a mechanism and $e$ be a Bayesian Nash equilibrium in the game. The pair $(G, e)$ is said to implement $k \in \mathbb{R}$ if the value of (1) is $k$ in $G$ if players follow the equilibrium strategies specified by $e$. Notice that $e$ can be assumed to be a pure strategy equilibrium without loss of generality. This is because one can always define a game corresponding to $G$ where the pure strategies in the new game are the mixed strategies in $G$. However, chance may randomize when taking an action. (In fact, we will show that the optimal mechanism generically involves randomization.) The next lemma states that we can restrict attention to a particularly small class of voting games.

Lemma 2 Suppose that $(G, e)$ implements $k(\in \mathbb{R})$. Then there exists a game $G^{\prime}$ and an equilibrium $e^{\prime}$ in $G^{\prime}$ that also implements $k$, with the following properties:

(i) the game $G^{\prime}$ is a sequential one,

(ii) at any information set in $G^{\prime}$, the possible actions of the voters are reporting signal $\mathcal{H}$ and reporting signal $\mathcal{L}$,

(iii) for each voter, there is a single information set in $G^{\prime}$ where she has to take an action, and

(iv) whenever a certain voter has to take an action at a certain information set in $e^{\prime}$, the voter acquires information and reports it truthfully.

Notice that (i)-(iii) are attributes of the game $G^{\prime}$ and (iv) is a property of the equilibrium $e^{\prime}$. The lemma above claims that we can restrict attention to a very simple class of games: The SP randomly orders the voters and asks them sequentially to acquire information and report it to him. After asking a voter, the SP decides whether to take an action or to ask an additional voter. Notice that (iii) says no information is revealed to the voters. ${ }^{2}$ When a voter is asked to collect information, she does not know her position in the sequence, and she does not know what other voters reported to the SP. Although the proof of this lemma is easy, the lemma is the heart of the paper and enables us to analyze the optimal voting scheme.

Proof. Suppose that $(G, e)$ implements $k$. Let us modify $G$ in the following ways:

(a) Rearrange the information sets such that the players move one after the other.

(b) If, at some information set, a certain voter does not acquire information but takes an action in $e$, then modify the game such that chance moves at that information set and takes the same action as the voter took in $(G, e)$.

to ask more voters to collect information, to reveal some information to certain voters, or to make a final decision. It can be useful to think of the chance as a mediator or a machine as in Myerson (1986).

${ }^{2}$ Myerson (1986) claims a similar result for a more general class of multistage games with communication. He claims (see page 339 in Myerson (1986)) that there is no loss of generality in assuming that, at each stage, the players report their new information. Furthermore, the SP only recommends actions to the players. Smorodinsky and Tennenholtz (2003) also arrive at similar conclusions. 
(c) If, in $G$, some voter takes an action other than reporting $\mathcal{H}$ or $\mathcal{L}$, modify the game such that the voter reports her information and chance takes the corresponding action.

(d) After change (b), a voter always acquires information no matter at which information set she has to take an action. So, unify all of the information sets where a certain voter has to take an action.

Notice that, after applying (a)-(d), we defined a new game $G^{\prime}$ and a strategy profile $e^{\prime}$ such that $\left(G^{\prime}, e^{\prime}\right)$ satisfies (i)-(iv). Obviously $\left(G^{\prime}, e^{\prime}\right)$ implements $k$, too. It remains to show that in $G^{\prime}$, the strategy profile $e^{\prime}$ is indeed an equilibrium. But if there were a profitable deviation, then by (c), there would have been a profitable deviation in $e$ too.

One can assume that the optimal mechanism is ex-ante symmetric with respect to $\mathcal{H}(H)$ and $\mathcal{L}(L)$. The reason is the following. Let us assume that the optimal mechanism is asymmetric. Then let us consider another mechanism where the role of $\mathcal{H}(H)$ and $\mathcal{L}(L)$ are switched. This is clearly also an optimal mechanism. Now consider the mechanism in which the SP uses the previous two mechanisms, each with probability one-half. This mechanism is also optimal, and it is ex-ante symmetric with respect to $\mathcal{H}$ and $\mathcal{L}$.

One can also assume that the SP orders the individuals uniformly and asks them to report their signals according to the realized order. (The voters do not know the realization of the order.)

From now on, we restrict attention to mechanisms that satisfy (i)-(iv) in Lemma 2 and are symmetric with respect to $\mathcal{H}(H)$ and $\mathcal{L}(L)$. Furthermore, the voters are asked according to the uniform ordering.

\section{Incentive Compatibility}

The goal of this subsection is to explicitly characterize the incentive compatibility constraint, that is, the constraint that guarantees the voters indeed have incentive to collect information when asked instead of just reporting something. (Another incentive compatibility constraint guarantees that a voter, upon acquiring information, will report her signal truthfully. As we will show, that constraint is trivially satisfied.) The derivation of this constraint is somewhat different from the usual incentive constraint in Bayesian mechanism design, since the voters do not have private information to start with. The idea of deriving this constraint is to compare a voter's expected payoff if she acquires information and reports it truthfully with her payoff if she just randomizes between reporting signal $\mathcal{H}$ and reporting signal $\mathcal{L}$ without actually drawing a signal. Surprisingly, the difference between these two payoffs can be expressed in a fairly simple form.

Let us fix a voting mechanism $M$. Let $\bar{L}$ denote the expected length of a sequence of signals after which the SP stops and makes a decision, conditional on each voter acquiring information upon being asked. That is, $\bar{L}$ is the expected number of voters asked in $M$. Let $S(K)$ denote the set of signal sequences that are weakly shorter than $K$. Let $s$ be a finite sequence of signal realizations. Let $l(s)$ denote the length of this sequence. The mechanism may involve some randomization by the SP. Let $r(s, M a j)(r(s, M i n))$ denote the probability that the SP stops the mechanism after 
the sequence $s$ (but not before) and makes the majority (minority) decision, conditional on $s$ being realized. (A majority (minority) decision after a sequence $s$ means taking action $a_{H}$ if and only if there are more (fewer) signals $\mathcal{H}$ in $s$ than signal $\mathcal{L}$.) Notice that the randomization of the SP is independent of the realization of the sequence. (All of the randomizations can be made ex-ante.) Let $p(s)$ denote the unconditional probability of the sequence $s$ and $p_{c}(s)$ the probability of $s$ conditional on being asked. Let $d(s)$ denote $\# \mathcal{H}(s)-\# \mathcal{L}(s)$. Hence the payoff of a voter who has been asked to collect information is

$$
\sum_{s \in S(N)} p_{c}(s)(r(s, M a j) B(|d(s)|)+r(s, \text { Min }) R(|d(s)|))-c
$$

Take a sequence $s$ and its complement $\bar{s}$. (Complement means that $\mathcal{H}$ and $\mathcal{L}$ are reversed. For example, if $s=(\mathcal{H}, \mathcal{H}, \mathcal{L})$, then $\bar{s}=(\mathcal{L}, \mathcal{L}, \mathcal{H})$.) Suppose that, in this sequence, there are $i+d$ signals $\mathcal{H}$ and $i$ signals $\mathcal{L}$ (where $d, i \geq 0$ ). Furthermore, suppose that the action of the SP is $a_{H}$ after the sequence $s$ and $a_{L}$ after the sequence $\bar{s}$. (That is, the SP makes the majority decision.) The probabilities of these sequences are

$$
\begin{aligned}
p(s) & =p(H) p(s \mid H)+p(L) p(s \mid L) \\
& =\frac{1}{2} p^{i+d} q^{i}+\frac{1}{2} q^{i+d} p^{i}=\frac{1}{2}(p q)^{i}\left(p^{d}+q^{d}\right), \\
p(\bar{s}) & =p(H) p(\bar{s} \mid H)+p(L) p(\bar{s} \mid L) \\
& =\frac{1}{2}(p q)^{i+d}\left(p^{-d}+q^{-d}\right) .
\end{aligned}
$$

Furthermore, these probabilities conditional on being asked are

$$
\begin{aligned}
& p_{c}(s)=\frac{2 i+d}{\bar{L}} \frac{1}{2}(p q)^{i}\left(p^{d}+q^{d}\right), \\
& p_{c}(\bar{s})=\frac{2 i+d}{\bar{L}} \frac{1}{2}(p q)^{i+d}\left(p^{-d}+q^{-d}\right) .
\end{aligned}
$$

The previous equations used Bayes' formula,

$$
p(A \mid B)=\frac{p(A \cap B)}{p(B)},
$$

where $A$ is the set of events where sequence $s$ is realized, and $B$ is the set of events where the deviator is asked to acquire information. Since the SP orders the voters independently of the realizations of the signals,

$$
p(A \cap B)=p(s) \frac{2 i+d}{N},
$$

where $(2 i+d) / N$ is the probability that the deviator is asked to report a signal if a decision is made after a sequence with length $2 i+d$. The unconditional probability that the deviator is asked, $p(B)$, is clearly $\bar{L} / N$.

Since the mechanism is symmetric with respect to $\mathcal{H}$ and $\mathcal{L}$, one can assume that the deviator always reports $\mathcal{H}$. Next, we compute the conditional probabilities of $s$ and $\bar{s}, p^{\prime}(s)$ and $p^{\prime}(\bar{s})$, given 
that the deviator always reports $\mathcal{H}$.

$$
\begin{aligned}
& p_{c}^{\prime}(s)=\frac{i+d}{\bar{L}} \frac{1}{2}(p q)^{i}\left(p^{d-1}+q^{d-1}\right)=\frac{i+d}{2 i+d} p_{c}(s) \frac{p^{d-1}+q^{d-1}}{p^{d}+q^{d}} \\
& p_{c}^{\prime}(\bar{s})=\frac{i}{\bar{L}} \frac{1}{2}(p q)^{i+d}\left(p^{-d-1}+q^{-d-1}\right)=\frac{i}{2 i+d} p_{c}(\bar{s}) \frac{p^{-d-1}+q^{-d-1}}{p^{-d}+q^{-d}} .
\end{aligned}
$$

This is because the probability that the deviator is asked is $(i+d) / \bar{L}$ conditional on $s$ being realized. Furthermore, the deviator knows that one of the signals $\mathcal{H}$ in sequence $s$ (or $\bar{s}$ ) is due to her deviation and has nothing to do with the distribution of the signals. Hence, when she computes the probability of a sequence, she takes away a signal $\mathcal{H}$ and computes the likelihood of the remainder of the sequence. Notice that by Lemma 1 ,

$$
\begin{aligned}
B(d-1) \frac{p^{d-1}+q^{d-1}}{p^{d}+q^{d}} & =\frac{B(d)}{p}, \\
B(d+1) \frac{p^{-d-1}+q^{-d-1}}{p^{-d}+q^{-d}} & =\frac{B(d)}{q} .
\end{aligned}
$$

If $s$ is realized and the deviator reported $\mathcal{H}$, then her expected utility from the majority decision is $B(d-1)$. (Again, this is because the deviator computes the posterior as if there were one fewer signal $\mathcal{H}$ in $s$.) Similarly if $\bar{s}$ is realized, her expected utility is $B(d+1)$. Hence, if the SP makes the majority decision after $s$ and $\bar{s}$, the difference between the expected benefit of the voter when she collects information and when she does not, conditional on being asked and on the realization of $s$ or $\bar{s}$, multiplied by the conditional probabilities of $s$ and $\bar{s}$, is:

$$
\begin{aligned}
& p(s) B(d)\left[\frac{2 i+d}{\bar{L}}-\frac{i+d}{p \bar{L}}\right]+p(\bar{s}) B(d)\left[\frac{2 i+d}{\bar{L}}-\frac{i}{q \bar{L}}\right] \\
= & p(s) B(d)\left[2 \frac{2 i+d}{\bar{L}}-\frac{i+d}{p \bar{L}}-\frac{i}{q \bar{L}}\right] \\
= & p(s) B(d)\left[2 \frac{l(s)}{\bar{L}}-\frac{l(s)+d}{2 p \bar{L}}-\frac{l(s)-d}{2 q \bar{L}}\right] \\
= & p(s) \frac{B(d)}{\bar{L}}\left[-l(s) \frac{(p-q)^{2}}{2 p q}+d \frac{p-q}{2 p q}\right] \\
= & p(s) \frac{B(d)}{\bar{L}} \frac{p-q}{2 p q}[d-(p-q) l(s)] .
\end{aligned}
$$

(The first equality follows from $p(s)=p(\bar{s})$ and the second one from (2), (3) and (4).) What happens if, after the realization of $s$ and $\bar{s}$, the SP makes the minority decision? In this case, from Lemma 1,

$$
\begin{aligned}
R(d-1) \frac{p^{d-1}+q^{d-1}}{p^{d}+q^{d}} & =\frac{R(d)}{q}, \\
R(d+1) \frac{p^{-d-1}+q^{-d-1}}{p^{-d}+q^{-d}} & =\frac{R(d)}{p} .
\end{aligned}
$$


Hence

$$
\begin{aligned}
& p(s) R(d)\left[2 \frac{2 i+d}{\bar{L}}-\frac{i+d}{p \bar{L}}-\frac{i}{q \bar{L}}\right] \\
= & p(s) R(d)\left[2 \frac{l(s)}{\bar{L}}-\frac{l(s)+d}{2 q \bar{L}}-\frac{l(s)-d}{2 p \bar{L}}\right] \\
= & p(s) \frac{R(d)}{\bar{L}}\left[-l(s) \frac{(p-q)^{2}}{2 p q}-d \frac{p-q}{2 p q}\right] \\
= & p(s) \frac{R(d)}{\bar{L}} \frac{p-q}{2 p q}[-d-(p-q)(2 i+d)] .
\end{aligned}
$$

To compute the difference between the expected benefit of a voter who acquires information and a voter who just reports signal $\mathcal{H}$, one has to execute the following computation. First, multiply (5) by the probability of making the majority decision after the sequence $s, r(s, M a j)$. Then multiply (6) by the probability of making the minority decision after the sequence $s, r(s$, Min). Finally, sum up for all possible sequences of signals. A mechanism is incentive compatible if this difference exceeds the cost of information acquisition. Hence, the mechanism is incentive compatible if and only if

$$
\begin{aligned}
& \frac{p-q}{4 p q} \sum_{s \in S(N)} r(s, M a j) p(s) B(|d|)[|d(s)|-(p-q) l(s)] \\
& +\frac{p-q}{4 p q} \sum_{s \in S(N)} r(s, \text { Min }) p(s) R(|d|)[-|d(s)|-(p-q) l(s)] \\
\geq & c \bar{L}
\end{aligned}
$$

(Notice that $(p-q)$ is divided by $4 p q$ instead of $2 p q$ because in each term of the summation we have counted a sequence and its complement.)

So far, we have ignored the constraint that guarantees that once a voter acquires information, she indeed reports the trues signal. We show, however, that this constraint is satisfied whenever (7) is satisfied.

Remark 1 Suppose that (7) is satisfied. Then the voters have incentive to report the true signals.

Proof. Let $\bar{u}$ denote the probability of making the correct final decision, conditional on a voter being asked and reporting a signal corresponding to the true state of the world and the rest of the voters acquiring information and reporting it truthfully. Let $\underline{u}$ denote the probability of making the correct final decision, conditional on a voter being asked and reporting a signal not corresponding to the true state of the world and the rest of the voters acquiring information. Since the probability that a signal corresponds to the true state of the world is $p$, the incentive compatibility constraint, (7), can be rewritten as

$$
p \bar{u}+(1-p) \underline{u}-c \geq \frac{1}{2}(\bar{u}+\underline{u}) .
$$


Since $c>0$ and $p>1 / 2$ the previous inequality implies $\bar{u}>\underline{u}$, and in turn

$$
p \bar{u}+(1-p) \underline{u} \geq p \underline{u}+(1-p) \bar{u} .
$$

This is exactly the constraint that guarantees that a voter reports the true signal if she acquires one.

Let us define $V(l, d)$ as follows:

$$
\{s \mid l(s)=l, d(s)=d\} .
$$

That is, $V(l, d)$ is the set of signal sequences of length $l$ in which the difference between the number of signals $\mathcal{H}$ and the number of signals $\mathcal{L}$ is exactly $d$. We refer to $V(l, d)$ as a state. Call a state $V(l, d)$ feasible if $l \leq N,|d| \leq l$ and $l-d$ is divisible by two. Let $\mathcal{V}(N)$ denote the set of feasible states. We can extend the definition of the functions $d$ and $l$ to these states. That is, if $V \in \mathcal{V}(N)$, then $d(V)=d$ and $l(V)=l$ if and only if $V=V(l, d)$.

Three decisions can be made at any state: the majority decision $(\mathrm{Maj})$, the minority decision $(M i n)$, and the decision to ask one more voter $(C)$. The mechanism determines the probabilities over these decisions at each state. What the mechanism specifies after reaching $V(l, d)$ may depend on the history, that is, on how $V(l, d)$ is actually reached. However, for each mechanism, there exists another one that operates as follows: The decision at $V(l, d)$ depends only on the probabilities determined by the original mechanism, not on the actual sequence. (For example, the probability of making the majority decision conditional on being at state $V(l, d)$ is $\sum_{s \in V(l, d)} r(s, M a j)$.) Notice that the unconditional probability of any sequence in a certain state is the same. Hence, from (7), it is clear that if the original mechanism was incentive compatible, the new one is also incentive compatible. The value of the objective function is also the same, so one can restrict attention to those mechanisms where the decision at each state is independent of the history.

Definition 1 A mechanism is said to be Markovian if for all states $V \in \mathcal{V}(N)$, the decision $D(\in\{M a j, M i n, C\})$ at $V$ depends only on $l(V)$ and $d(V)$.

From now on, we restrict attention to Markovian mechanisms. Let $M$ denote a mechanism. Let $p(V, D, M)$ (where $D \in\{M a j, M i n, C\}, V \in \mathcal{V}(N)$ ) denote the probability of making decision $D$ at state $V$, conditional on $M$ and being at state $V$. A Markovian mechanism $M$ can be identified by the collection of these probabilities, that is, by

$$
\{p(V, D, M) \mid D \in\{M a j, \operatorname{Min}, C\}, V \in \mathcal{V}(N)\} .
$$

Define $\rho(V, D, M)(V \in \mathcal{V}(N), D \in\{$ Maj, Min, $C\})$ as the probability of reaching state $V$ and making a decision $D$ given mechanism $M$. Furthermore, let $p(V, M)$ denote the probability of reaching $V$ conditional on $M$. Then clearly

$$
p(V, D, M)=\frac{\rho(V, D, M)}{p(V, M)} .
$$


With this notation, we can rewrite the maximization problem of the SP and (7) as follows:

$$
\max _{M} N \sum_{V \in \mathcal{V}(N)}[\rho(V, M a j, M) B(|d(V)|)+\rho(V, \text { Min }, M) R(|d(V)|)]-c \bar{L}(M),
$$

subject to the incentive compatibility constraint

$$
\begin{aligned}
& \frac{p-q}{4 p q} \sum_{V \in \mathcal{V}(N)} \rho(V, M a j, M) B(|d(V)|)[|d(V)|-(p-q) l(V)] \\
& +\frac{p-q}{4 p q} \sum_{V \in \mathcal{V}(N)} \rho(V, \operatorname{Min}, M) R(|d(V)|)[-|d(V)|-(p-q) l(V)] \\
\geq & c \bar{L}(M),
\end{aligned}
$$

where $\bar{L}(M)$ is the expected number of voters asked in mechanism $M$. The left side of the last inequality is basically the probability of some voter being pivotal times $\bar{L}(M)$.

Suppose that a mechanism $M$ is a probability mixture of some incentive compatible mechanisms $M_{1}, \ldots, M_{n}$. That is, the SP uses mechanism $M_{i}$ with probability $p_{i}$. (The voters only know $p_{i}$ 's, but not the realization of the randomization.) Then

$$
\rho(V, D, M)=\sum_{i=1}^{n} p_{i} \rho\left(V, D, M_{i}\right)
$$

and clearly

$$
\bar{L}(M)=\sum_{i=1}^{n} p_{i} \bar{L}\left(M_{i}\right) .
$$

Notice that both (9) and (10) are linear in $\rho(V, D, M)$. Hence we can claim the following:

Remark 2 The probability mixture of incentive compatible mechanisms is also incentive compatible. Furthermore, the value of the objective function is the probability mixture of the values of the objective functions corresponding to the mechanisms used in the mixture.

Next, we show that in optimal mechanisms, the voters are indifferent between collecting information and making a random report.

Lemma 3 Suppose that the first-best mechanism is not incentive compatible and $M^{*}$ is either the ex-ante optimal mechanism or the ex-ante optimal mechanism among the ex-post efficient ones. Then (10) holds with equality.

Proof. See Appendix A.

\section{Continuation Mechanisms}

The arguments of most proofs regarding optimality of mechanisms involve modifying the mechanism at some states. We introduce the notion of a continuation mechanism, which is the part of 
a mechanism that follows after reaching a certain state. In the first-best mechanism, when the SP had to decide whether to ask an additional voter or to make a decision, he only had to compare the cost of a continuation mechanism from a particular state with the expected increase in precision due to the continuation mechanism. It did not matter how the mechanism would continue starting from another state. Such a simple argument cannot be used when the mechanism is subject to the incentive compatibility constraint. When the scheme must be incentive compatible, there is an interaction between different continuation mechanisms. In particular, when the SP decides whether to employ a certain continuation mechanism at a certain state, he faces a trade-off: Employing the continuation mechanism can make it impossible to employ another continuation mechanism at a different state because of the incentive compatibility constraint. Hence, finding an optimal incentive compatible voting scheme is not a stochastic dynamic programming problem.

Recall, the reason why the function defining the first-best scheme was decreasing (see Proposition 1 ) is that the more voters have already been asked, the less valuable it is to ask an additional voter given a certain posterior. In what follows, we establish a similar monotonicity property of continuation mechanisms, even when the schemes are subject to the incentive compatibility constraint, so long as we also require that decisions be ex-post efficient. To that end, we define the efficiency of continuation mechanisms. Then, we show that the efficiency of a given continuation mechanism decreases in the number of voters already asked. In addition, we prove that in the optimal ex-post efficient mechanism, if some continuation mechanism is employed with positive probability, any more efficient continuation mechanism that is also feasible must also be employed.

A continuation mechanism at state $V(l, d)$ is just a mechanism $M$ defined by (8) specifying the decisions of the SP after reaching $V(l, d)$. Let $M(l, d)$ denote the continuation mechanism $M$ at $V(l, d)$. Similarly to $\rho(V, D, M)$, one can define $\rho^{d}(V, D, M(l, d))$ as the probability of reaching $V$ from $V(l, d)$ and making a decision $D$ given continuation mechanism $M$. However, a majority (minority) decision, $M a j(M i n)$, means taking action $a_{H}$ if and only if $d(V)+d>(<) 0$. A continuation mechanism $M$ at $V(l, d)$ is said to be ex-post efficient if

$$
\rho^{d}(V, \operatorname{Min}, M(l, d))=0 \text { for all } V \in \mathcal{V}(N) .
$$

That is, the minority decision is never made. Notice that whether a continuation mechanism is ex-post efficient depends on the state $V$ at which it is employed. However, it depends only on $d(V)$ and not on $l(V)$. Hence, an ex-post efficient continuation mechanism at $V(l, d)$ is necessarily asymmetric with respect to $\mathcal{H}(H)$ and $\mathcal{L}(L)$ unless $d=0$.

For an example of a continuation mechanism, consider $M$ defined by

$$
p(V(1,1), M a j, M)=p(V(1,-1), M a j, M)=1 .
$$

That is, $M$ specifies action $a_{H}$ after signal $\mathcal{H}$ and action $a_{L}$ after signal $\mathcal{L}$. Suppose that $M$ is used at $V(3,3)$. Then,

$$
\rho^{3}(V(4,4), \operatorname{Maj}, M(3,3))=p(3), \rho^{3}(V(4,2), \operatorname{Min}, M(3,3))=q(3),
$$


and all the other probabilities are zero.

Definition $2 A$ continuation mechanism $M$ is feasible at $V(l, d)$ with respect to a mechanism $M^{\prime}$ if (i) $p\left(V(l, d), M^{\prime}\right)>0$, and (ii) if $p(V, M)>0$ then $l(V) \leq N-l$.

The previous definition says that given a mechanism $M^{\prime}$, a continuation mechanism is feasible at $V(l, d)$ if and only if $V(l, d)$ is indeed reached with positive probability according to $M^{\prime}$. Furthermore, the continuation mechanism $M$ never specifies that the SP asks more voters than the number available after reaching $V(l, d)$, that is $N-l$.

Let $d \operatorname{Obj}(M(l, d))$ denote the change in the objective function of the SP if, instead of making the majority decision at $V(l, d)$, the mechanism continues according to $M$ conditional on being at $V(l, d)$. That is,

$$
\begin{aligned}
\operatorname{dObj}(M(l, d))= & N \sum_{V \in \mathcal{V}(N)} \rho^{d}(V, M a j, M) B(|d(V)+d|) \\
& +N \sum_{V \in \mathcal{V}(N)} \rho^{d}(V, M i n, M) R(|d(V)+d|) \\
& -c \bar{L}(M)-N B(|d|),
\end{aligned}
$$

where $\bar{L}_{d}(M)$ denotes the expected number of voters asked according to $M$ if $M$ is employed at $V(l, d)$. Furthermore, define $d I C(M(l, d))$ as

$$
\begin{aligned}
& \frac{p-q}{4 p q} \sum_{V \in \mathcal{V}(N)} \rho^{d}(V, M a j, M) B(|d(V)+d|)[|d(V)+d|-(p-q)(l(V)+l)] \\
& +\frac{p-q}{4 p q} \sum_{V \in \mathcal{V}(N)} \rho^{d}(V, M i n, M) R(|d(V)+d|)[-|d(V)+d|-(p-q)(l(V)+l)] \\
& -c \bar{L}_{d}(M)-\frac{p-q}{4 p q} B(|d|)[|d|-(p-q) l] .
\end{aligned}
$$

Roughly, $d I C(M(l, d))$ is the change in the incentive compatibility constraint if the SP continues asking voters according to $M$ instead of making the majority decision at $V(l, d)$ conditional on being at $V(l, d)$.

Suppose that for a mechanism $M^{\prime}, \rho\left(V(l, d), M a j, M^{\prime}\right)>0$. Then, if $M^{\prime}$ is modified such that the SP continues asking additional voters according to $M$ at $V(l, d)$ instead of making the majority decision, the value of the SP's objective function increases by $\rho\left(V(l, d), M a j, M^{\prime}\right) d O b j(M(l, d))$. Furthermore, the new incentive compatibility constraint is almost the same as the one corresponding to $M^{\prime}$, but $\rho\left(V(l, d), M a j, M^{\prime}\right) d I C(M(l, d))$ must be added to the left side of (10). Notice that neither $\operatorname{dObj}(M(l, d))$ nor $d I C(M(l, d))$ depends on $M^{\prime}$. This is why one can evaluate the efficiency of a continuation mechanism independent of $M^{\prime}$.

Definition 3 Let $M$ be a mechanism. The efficiency of $M$ at $V(l, d)$ is defined as

$$
e(M(l, d))=\left|\frac{d \operatorname{Obj}(M(l, d))}{d I C(M(l, d))}\right| .
$$


The efficiency of a continuation mechanism essentially says how much the objective function of the SP increases if the incentive compatibility constraint changes by one unit. There are continuation mechanisms for which $d \operatorname{Obj}(M(l, d))$ as well as $d I C(M(l, d))$ are negative, but those continuation mechanisms are obviously never employed in optimal mechanisms. There may also exist continuation mechanisms for which $d \operatorname{Obj}(M(l, d))$ and $d I C(M(l, d))$ are positive, but then, at $V(l, d)$ the SP always continues to ask more voters. The subjects of our interest are those continuation mechanisms for which $d \operatorname{Obj}(M(l, d))$ is positive but $d I C(M(l, d))$ is negative, or vice verse. That is, employing $M$ at $V(l, d)$ increases the value of the SP's objective function at the cost of incentive compatibility.

Lemma 4 Let $M$ be a feasible ex-post efficient continuation mechanism at $V(l, d)$. Then

$$
\sum_{V \in \mathcal{V}(N)} \rho^{d}(V, M a j, M(l, d)) B(|d(V)+d|) \geq B(|d|) .
$$

The right side of the inequality is the SP's posterior at $V(l, d)$ about the true state of the world. The left side is the expected posterior if continuation mechanism $M$ is used. The lemma claims that the expected posterior at $V$ if an ex-post efficient continuation mechanism is employed at $V$ is at least as precise as the posterior at $V$. Clearly, more information cannot result in a less accurate expected posterior.

Proof. See Appendix A.

The next lemma shows the following. Suppose that a mechanism $M$ is ex-post efficient at a state $V$ if $d(V)=d$ and that $V^{\prime}$ is also a state for which $d\left(V^{\prime}\right)=d$. Then $M$ is more efficient at $V^{\prime}$ than at $V$ if $l(V)>l\left(V^{\prime}\right)$. This lemma is essential for characterizing the optimal ex-post efficient mechanism.

Lemma 5 Let $M$ be a mechanism such that $M$ is ex-post efficient at $V$ if $d(V)=d$. Suppose that $M$ is feasible at $V(l, d)$ as well as at $V\left(l^{\prime}, d\right)$. If $l>l^{\prime}$, then

$$
e(M(l, d))<e\left(M\left(l^{\prime}, d\right)\right) .
$$

Proof. Because of Lemma 1, the probability of realizing a certain sequence conditional on being at $V(l, d)$ is the same as that conditional on being at $V\left(l^{\prime}, d\right)$. Hence, $\rho^{d}(V, D, M(l, d))=$ $\rho^{d}\left(V, D, M\left(l^{\prime}, d\right)\right)$ for all $V \in \mathcal{V}(N)$ and $D \in\{M a j, M i n, C\}$. From (11), it follows that

$$
\operatorname{dObj}(M(l, d))=\operatorname{dObj}\left(M\left(l^{\prime}, d\right)\right) .
$$

Furthermore, from (12),

$$
\begin{aligned}
& d I C(M(l, d))-d I C\left(M\left(l^{\prime}, d\right)\right) \\
= & -\left(l-l^{\prime}\right) \frac{(p-q)^{2}}{4 p q}\left[\sum_{V \in \mathcal{V}(N)} \rho^{d}(V, M a j, M(l, d)) B(|d(V)+d|)-B(|d(V)|)\right] .
\end{aligned}
$$


From the previous lemma, we know that the term in square brackets is positive. Since $l>l^{\prime}$, this expression is negative. Hence $e(M(l, d))<e\left(M\left(l^{\prime}, d\right)\right)$.

Given a mechanism $M^{\prime}$ and a feasible continuation mechanism $M$ at $V(l, d)$ with respect to $M^{\prime}$, let $\operatorname{Pr}\left(M(l, d) \mid M^{\prime}\right)$ denote the probability of using continuation mechanism $M$ according to $M^{\prime}$ conditional on reaching $V(l, d)$.

Lemma 6 Let $M^{*}$ be an optimal mechanism among the ex-post efficient ones. Suppose that $M$ and $M^{\prime}$ are feasible ex-post efficient continuation mechanisms with respect to $M^{*}$ at $V(l, d)$ and $V\left(l^{\prime}, d^{\prime}\right)$, respectively. Furthermore,

$$
\begin{aligned}
\operatorname{dObj}(M(l, d)) d I C(M(l, d)) & <0, \\
\operatorname{dObj}\left(M^{\prime}\left(l^{\prime}, d^{\prime}\right)\right) d I C\left(M^{\prime}\left(l^{\prime}, d^{\prime}\right)\right) & <0 .
\end{aligned}
$$

Suppose that $e(M(l, d))>e\left(M^{\prime}\left(l^{\prime}, d^{\prime}\right)\right)$ and $\operatorname{Pr}\left(M^{\prime}\left(l^{\prime}, d^{\prime}\right) \mid M^{*}\right)>0$. Then $p\left(V(l, d), C, M^{*}\right)=$ 1.

This lemma says that if $M^{*}$ is an optimal ex-post efficient mechanism, then it never happens that the mechanism stops at $V(l, d)$ with positive probability if there exists a feasible continuation mechanism at $V(l, d)$ that is more efficient than another continuation mechanism that is used with positive probability.

Proof. We only prove the lemma for the case when

$$
d I C(M(l, d)), d I C\left(M^{\prime}\left(l^{\prime}, d^{\prime}\right)\right)<0 .
$$

The proofs of the other cases are essentially identical to this one.

Suppose that the hypothesis of the lemma is satisfied but $p\left(V(l, d), C, M^{*}\right)<1$. Let us consider the following mechanism, denoted by $M^{* *} . M^{* *}$ is almost identical to $M^{*}$, only it is modified at $V(l, d)$ and $V\left(l^{\prime}, d^{\prime}\right)$. At $V(l, d)$, it uses the continuation mechanism $M$ with probability $\varepsilon(>0)$ instead of making the majority decision, and at $V\left(l^{\prime}, d^{\prime}\right)$ it stops with probability

$$
\varepsilon \frac{d I C(M(l, d)) p\left(V(l, d), M^{*}\right)}{d I C\left(M^{\prime}\left(l^{\prime}, d^{\prime}\right)\right) p\left(V\left(l^{\prime}, d^{\prime}\right), M^{*}\right)}
$$

and makes the majority decision instead of using $M^{\prime}$ as a continuation mechanism. If $\varepsilon$ satisfies

$$
\varepsilon<\min \left\{p\left(V(l, d), M a j, M^{*}\right), \operatorname{Pr}\left(M^{\prime}\left(l^{\prime}, d^{\prime}\right) \mid M^{*}\right) \frac{d I C\left(M\left(l^{\prime}, d^{\prime}\right)\right) p\left(V\left(l^{\prime}, d^{\prime}\right), M^{*}\right)}{d I C(M(l, d)) p\left(V(l, d), M^{*}\right)}\right\},
$$

these changes are indeed feasible and the new mechanism is well-defined. (Since $p\left(V(l, d), C, M^{*}\right)<$ $1, \varepsilon$ can be chosen to be bigger than zero.) The change in the incentive compatibility constraint is

$$
\begin{aligned}
& p\left(V(l, d), M^{*}\right) \varepsilon d I C(M(l, d)) \\
& -p\left(V\left(l^{\prime}, d^{\prime}\right), M^{*}\right) \varepsilon \frac{d I C(M(l, d)) p\left(V(l, d), M^{*}\right)}{d I C\left(M^{\prime}\left(l^{\prime}, d^{\prime}\right)\right) p\left(V\left(l^{\prime}, d^{\prime}\right), M^{*}\right)} d I C\left(M^{\prime}\left(l^{\prime}, d^{\prime}\right)\right) \\
= & 0 .
\end{aligned}
$$


Hence, since the mechanism $M^{*}$ is incentive compatible, the new mechanism is also incentive compatible. Furthermore, the change in the objective function of the SP is

$$
\begin{aligned}
& p\left(V(l, d), M^{*}\right) \varepsilon d \operatorname{Obj}(M(l, d)) \\
& -p\left(V\left(l^{\prime}, d^{\prime}\right), M^{*}\right) \varepsilon \frac{d I C(M(l, d)) p\left(V(l, d), M^{*}\right)}{d I C\left(M^{\prime}\left(l^{\prime}, d^{\prime}\right)\right) p\left(V\left(l^{\prime}, d^{\prime}\right), M^{*}\right)} \operatorname{Oobj}\left(M^{\prime}\left(l^{\prime}, d^{\prime}\right)\right) \\
= & p\left(V(l, d), M^{*}\right) \varepsilon e(M(l, d))|I C(M(l, d))| \\
& -p\left(V\left(l^{\prime}, d^{\prime}\right), M^{*}\right) \varepsilon \frac{|d I C(M(l, d))| p\left(V(l, d), M^{*}\right)}{p\left(V\left(l^{\prime}, d^{\prime}\right), M^{*}\right)} e\left(M^{\prime}\left(l^{\prime}, d^{\prime}\right)\right) \\
= & p\left(V(l, d), M^{*}\right) \varepsilon|I C(M(l, d))|\left(e(M(l, d))-e\left(M^{\prime}\left(l^{\prime}, d^{\prime}\right)\right)\right) .
\end{aligned}
$$

Since $e(M(l, d))>e\left(M^{\prime}\left(l^{\prime}, d^{\prime}\right)\right)$, the new mechanism increases the value of the objective function, contradicting the optimality of $M^{*}$.

Notice that the only reason why $M(l, d)$ and $M^{\prime}\left(l^{\prime}, d^{\prime}\right)$ were required to be ex-post efficient is because $M^{*}$ was the optimal mechanism among the ex-post efficient one. The proof of the lemma has not used the ex-post efficiency property of the continuation mechanisms. Hence, we can claim a similar result for the optimal (not necessarily ex-post efficient) mechanism.

Remark 3 Let $M^{*}$ be an optimal mechanism. Suppose that $M$ and $M^{\prime}$ are feasible continuation mechanisms with respect to $M^{*}$ at $V(l, d)$ and $V\left(l^{\prime}, d^{\prime}\right)$, respectively. Furthermore,

$$
\begin{aligned}
\operatorname{dObj}(M(l, d)) d I C(M(l, d)) & <0, \\
\operatorname{dObj}\left(M^{\prime}\left(l^{\prime}, d^{\prime}\right)\right) d I C\left(M^{\prime}\left(l^{\prime}, d^{\prime}\right)\right) & <0 .
\end{aligned}
$$

Suppose that $e(M(l, d))>e\left(M^{\prime}\left(l^{\prime}, d^{\prime}\right)\right)$ and $\operatorname{Pr}\left(M^{\prime}\left(l^{\prime}, d^{\prime}\right) \mid M^{*}\right)>0$. Then $p\left(V(l, d), C, M^{*}\right)=$ 1.

Lemma 7 Suppose that $M^{*}$ is an ex-ante optimal, ex-post efficient mechanism and

$$
p\left(V(l, d), M^{*}\right) \operatorname{Pr}\left(M(l, d) \mid M^{*}\right)>0 .
$$

Then there exists a state $V^{\prime} \in \mathcal{V}(N-l)$ such that $d\left(V^{\prime}\right) d<0$ and

$$
\rho^{d}\left(V^{\prime}, M a j, M(l, d)\right)>0 .
$$

The condition $d\left(V^{\prime}\right) d<0$ means that the majority decision at $V^{\prime}$ is different from the majority decision at $V(l, d)$. Hence, the previous lemma says that if a continuation mechanism in an optimal mechanism is employed at state $V(l, d)$, then it reaches and specifies the majority decision in at least one state $V^{\prime}$ where the decision differs from the majority decision at $V(l, d)$. This essentially means that a voter is never asked to collect information if her vote will not affect the action taken by the Social Planner.

Proof. See Appendix A. 
Lemma 8 There exists an optimal mechanism $M^{*}$ among the ex-post efficient ones such that the SP either does not randomize or randomizes only at states $V(\widehat{l}, \widehat{d})$ and $V(\widehat{l},-\widehat{d})$. That is,

$$
p(V, C, M), p(V, M a j, M)>0 \Leftrightarrow l(V)=\widehat{l} \text { and }|d(V)|=\widehat{d} .
$$

Furthermore, generically, if the first-best mechanism is not incentive compatible, the optimal mechanism $M^{*}$ among the ex-post efficient ones must involve randomization.

Generically means that the measure of those $(p, c)$ pairs for which the statement of the lemma is not true has Lebesgue measure zero.

Proof. See Appendix A.

\section{Optimal Mechanisms}

In this section, we first characterize the optimal ex-post efficient mechanism. We show that it has very similar properties to the first-best voting scheme. Then we discuss some attributes of this mechanism. Finally, we show that the ex-ante optimal mechanism sometimes involves ex-post inefficient decisions.

\subsection{Optimal Ex-post Efficient Mechanism}

We are ready to characterize the optimal mechanism in the class of ex-post efficient mechanisms, that is, the class of mechanisms where the SP always makes a majority decision.

Theorem 1 Let $M^{\star}$ be an ex-ante optimal mechanism among the ex-post efficient ones. Then, there exists a decreasing step function $f: \mathbb{N}_{+} \rightarrow \mathbb{N}_{+}$such that

$$
\begin{aligned}
\text { for all } l & \in \mathbb{N}_{+}, f(l+1)=f(l) \text { or } f(l+1)=f(l)-1, \\
f(N) & =1 .
\end{aligned}
$$

Let

$$
W=\{V: f(l(V))=|d(V)|, f(l(V)-1)=f(l(V))+1\} .
$$

$M^{\star}$ is defined by the following three conditions:

$$
\begin{aligned}
\text { (i) if } V & \notin W \text { and } f(l(V)) \leq|d(V)| \text { then } p\left(V, M a j, M^{\star}\right)=1, \\
\text { (ii) if } V & \notin W \text { and } f(l(V))>|d(V)| \text { then } p\left(V, C, M^{\star}\right)=1, \\
\text { (iii) if } V & \in W \text { then } p\left(V, M a j, M^{\star}\right) \geq 0, p\left(V, C, M^{\star}\right)>0 .
\end{aligned}
$$

Furthermore, generically, there exists an optimal ex-post efficient mechanism for which there is only a single $V \in W$ such that $p\left(V, M a j, M^{\star}\right)>0$. 
Theorem 1 essentially claims that an optimal ex-post efficient mechanism can be described by the decreasing step function $f$. The SP keeps asking the voters sequentially to collect information and report it to him. Once a state $V(l, d)$ is reached where $f(l)=|d|$, the SP makes the majority decision. Since the function $f$ is decreasing, the more voters the SP has already asked, the less precise a posterior induces the SP to stop asking voters and take an action. Furthermore, the function $f$ never jumps down by more than one. Since, generically, in such a deterministic mechanism the incentive compatibility constraint holds with inequality, the SP randomizes at certain states. At these states, the SP makes the majority decision with some probability and asks an additional voter with some probability. If the voter confirms his posterior, he makes the majority decision; otherwise, he continues according to the rule specified by $f$. Property (iii) says randomization can happen at a state $V(l, d)$ only if $f(l)=|d|$ and the function $f$ jumps down at $l-1$. Notice that $f(N)=1$ does not imply that potentially all the voters are asked to acquire information. For example, if $f(K)=1$ and $K<N$, then the maximum number of voters acquiring information is less than $K+1$.

The proof of Theorem 1 is relegated to Appendix B. Here, however, we present the main argument of the proof. Let $\bar{d}$ denote the largest $d$ for which there exists an $l$ such that $p\left(V(l, d), M^{*}\right)>$ 0 . For each $d(\in\{1, \ldots, \bar{d}-1\})$, let $\alpha(d)$ denote the largest possible $l$ for which $p\left(V(l, d), C, M^{*}\right)$ is positive. (It can be shown that the function $\alpha$ is well defined.) We claim that, conditional on reaching $V(l, d)$, the mechanism continues at $V(l, d)$ if and only if $l<\alpha(d)$. If $l<\alpha(d)$, then the continuation mechanism at $V(\alpha(d), d)$ is feasible at $V(l, d)$, too. By Lemma 5 , the continuation mechanism used at $V(a(d), d)$ is more efficient at $V(l, d)$. Hence, from Lemma 6 , it is impossible that the mechanism stops at $V(l, d)$ conditional on reaching $V(l, d)$. (If $\alpha(d)<l$, then the mechanism stops at $V(l, d)$ by the definition of $\alpha$.) We show that $\alpha$ is a strictly decreasing function. Suppose not, and $d>d^{\prime}$ and $\alpha(d) \geq \alpha\left(d^{\prime}\right)$. This means that the mechanism surely continues at $V(\alpha(d), d)$. Notice, however, that after continuing from $V(\alpha(d), d)$, the mechanism must stop whenever the difference between the number of different signals is $d^{\prime}$. (This is because $\alpha\left(d^{\prime}\right)<\alpha(d)$.) Hence the decision of the SP after reaching $V(\alpha(d), d)$ is always the majority decision at this state, no matter what the voters report in the continuation mechanism. This contradicts the statement of Lemma 7. It can be shown that the mechanism continues at state $V(l, d) \in \mathcal{V}(N)$ if and only if $l \leq \alpha(d)$. Furthermore, since $\alpha$ is decreasing, $p\left(V(l, d), M a j, M^{*}\right)$ is positive if and only if $\alpha(d-1) \geq l-1$ and $\alpha(d)<l$. The first inequality guarantees that the mechanism continues at $V(l-1, d-1)$; hence $V(l, d)$ can indeed be reached. The second inequality guarantees that the mechanism does not continue at $V(l, d)$. The function $f$ in Theorem 1 can be defined as follows:

$$
f(l)=\min _{d \in\{1, \ldots, \bar{d}-1\}}\{d \mid \alpha(d) \leq l\} .
$$

Notice that $f(\alpha(d))=d$, so one can think of the function $f$ as the inverse of $\alpha$. Since $\alpha$ is strictly decreasing, the function $f$ is also decreasing. It is easy (but cumbersome) to show that the properties of $f$ described in the theorem hold. As we pointed out earlier, from Lemma 3 , it follows 
that the optimal mechanism generically involves randomization. From Lemma 6 and Lemma 7, it follows that randomization can happen only at states in $W$. From Lemma 8 , it follows that there generically exists an optimal mechanism in which randomization happens at a single state.

Finally, we claim that the optimal voting scheme described in Theorem 1 is essentially unique.

Theorem 2 The optimal ex-post efficient mechanism described in Theorem 1 is generically unique and involves randomization only at a single state.

Proof. See Appendix A.

The argument of the proof of Theorem 2 is as follows. First, we show that if, for a certain pair $(p, c)$, there are at least two different optimal mechanisms, then there exists an optimal mechanism that involves randomizations in at least two different states. By Lemma 6, it follows that there are at least two continuation mechanisms that have the same efficiency. Since there are only finitely many continuation mechanisms, if the optimal mechanism were not generically unique, there would exist two continuation mechanisms with the same efficiency for a positive measure of $(p, c)$. In the proof of Theorem 2, we show that this is impossible.

\subsection{Discussion of the Ex-post Optimal Mechanism}

\section{Infinitely Many Voters}

The properties of the function $f$ in Theorem 1 above are very similar to those of the function $g$ in Proposition 1 characterizing the first-best voting scheme. (In general, $g$ is much bigger than $f$.) Recall that the function $g$ was decreasing because, as the number of voters who have not yet collected information decreases, the value of asking more voters also decreases. It is tempting to think that the properties of $f$ can be explained similarly. This is partially true, since as the number of voters asked increases, the number of available continuation mechanisms decreases. However, an even more important reason is the following. The way to guarantee a large probability of being pivotal to the voters is to make decisions after signal sequences where the difference between the numbers of different signals is small. That is, the SP makes a decision after sequences that generate imprecise posteriors. We claim that providing a large probability of being pivotal is more efficient after long sequences than after short sequences. The rough intuition is as follows. Conditional on being asked, a voter believes that she is most likely to have been asked along a long sequence rather than a short one. When a voter computes the probability of a sequence $s$ conditional on being asked, she multiplies the unconditional probability by $l(s) / \bar{L}(M)$. The actual probability of a long sequence is small, and hence the effect of such a sequence on the objective function is small. However, since the conditional probability of such a sequence is large, the effect on the incentive compatibility constraint is also large. This is the reason why the function $f$ is decreasing: After a short sequence the Social Planner makes a decision only if his posterior is precise, but after a long sequence he makes a decision even if his posterior is fuzzy. 
To demonstrate that this is the reason for the properties of the function $f$, we modify the model such that there are infinitely many voters and the Social Planner wants to maximize

$$
A E u-c \bar{L},
$$

where $A$ is a large constant. (Otherwise the model is the same as before.) In this modified model, in the first-best mechanism the value of asking an additional voter does not depend on the number voters already asked. (To see that the problem of the SP is well-defined, notice that the $A E u$ is bounded by $A$, but $c \bar{L}$ is not bounded.) Hence the function $g$ that characterizes the first-best scheme is simply a constant. We claim the following:

Proposition 2 If there are infinitely many voters, the first-best mechanism is characterized by a constant $k \in \mathbb{N}$. The SP stops asking voters if and only if the difference between the numbers of different signals is at least $k$. Furthermore, as A goes to infinity, $k$ converges to infinity.

We show, however, that whenever the first best mechanism is not incentive compatible, the optimal ex-post efficient mechanism can be characterized almost exactly as in Theorem 1. First, we argue that sometimes the first-best scheme is indeed not incentive compatible. In particular, given $p$ and $c$, if $A$ is large enough, the first-best mechanism is not incentive compatible. We argue that for given $p$ and $c$, there always exists a $k_{0} \in \mathbb{N}$ such that the mechanism is not incentive compatible if the SP stops asking voters only if $|d| \geq k_{0}$. This is because as $k_{0}$ goes to infinity, the probability of being pivotal, and hence also the benefit from collecting information, goes to zero. On the other hand, the cost of acquiring information does not change. The previous proposition says that if $A$ is large enough, the first-best mechanism can be characterized by $k>k_{0}$; hence the mechanism is not incentive compatible.

Proposition 3 Suppose that there are infinitely many voters, and the first-best mechanism is not incentive compatible. Let $M^{\star}$ be an ex-ante optimal, ex-post efficient mechanism. Then, generically, there exists a decreasing function $f: \mathbb{N}_{+} \rightarrow \mathbb{N}_{+}$and a $\widehat{l}, \widehat{d}, K \in \mathbb{N}_{+}$, such that

$$
\begin{aligned}
\text { for all } l & \in \mathbb{N}_{+}, f(l+1)=f(l) \text { or } f(l+1)=f(l)-1, \\
f(K) & =1 \text { and } f(\hat{l})=\widehat{d}=f(\widehat{l}-1)-1 .
\end{aligned}
$$

$M^{\star}$ is defined by the following three conditions:

$$
\begin{aligned}
\text { (i) if } V & \neq V(\widehat{l}, \widehat{d}) \text { and } f(l(V)) \leq|d(V)| \text { then } p\left(V, M a j, M^{\star}\right)=1, \\
\text { (ii) if } V & \neq V(\widehat{l}, \widehat{d}) \text { and } f(l(V))>|d(V)| \text { then } p\left(V, C, M^{\star}\right)=1, \\
\text { (iii) if } V & =V(\widehat{l}, \widehat{d}) \text { then } p\left(V, M a j, M^{\star}\right), p\left(V, C, M^{\star}\right)>0 .
\end{aligned}
$$

The only difference between the statements of Theorem 1 and the previous proposition is that $K$ (the integer at which the value of $f$ is one) is endogenously determined in the proposition. Recall that throughout the proof of Theorem 1, we used the assumption that there are finitely 
many voters only to show that $f(N)=1$. Hence the only part of the statement of Theorem 3 we have to show is that there exists a $K \in \mathbb{N}$ such that $f(K)=1$. In other words, the maximum number of voters asked in the optimal mechanism is finite.

Proof. Let $M^{*}$ denote the optimal ex-post efficient mechanism. Suppose by contradiction that for all $k \in \mathbb{N}, f(k)>1$. Since $M^{*}$ is not the first-best mechanism, there must exist a state $V(l, d)$ and a feasible continuation mechanism $\bar{M}$ at $V(l, d)$ that is employed with zero probability in $M^{*}$ even though it increases the objective function of the SP. Since $f$ is decreasing and $f(k)>1$ for all $k \in \mathbb{N}$, there must exist a $\bar{K} \in \mathbb{N}$, and $\bar{k}>1$, such that whenever $k>\bar{K}, f(k)=\bar{k}$. This means there are continuation mechanisms that are employed with positive probability at states $V(k, d)$ for infinitely many $k^{\prime} s$. In particular, the mechanism $M^{\prime}$, which specifies the majority decision at $V(l, d)$ if and only if $d \geq \bar{k}-1$ or $d \leq-\bar{k}-1$, is employed at all feasible states $V(k, 1)$ whenever $k>\bar{K}$. From (12), the change in incentive compatibility, $d I C\left(M^{\prime}(k, 1)\right)$, is

$$
\begin{aligned}
& \frac{k(p-q)^{2}}{4 p q}\left[B(1)-\sum_{V \in \mathcal{V}(N)} \rho^{d}\left(V, M a j, M^{\prime}\right) B(|d(V)+1|)\right] \\
& +\frac{p-q}{4 p q} \sum_{V \in \mathcal{V}(N)} \rho^{d}\left(V, M a j, M^{\prime}\right) B(|1+d|)[|d(V)+1|-(p-q) l(V)] \\
& -c \bar{L}_{1}\left(M^{\prime}\right)-\frac{p-q}{4 p q} B(1) .
\end{aligned}
$$

From the proof of Lemma 4, it follows that the term in square brackets in the first line is negative. Hence, as $k$ goes to infinity, the term in the first line goes to minus infinity. Since the rest of the terms do not depend on $k$, one can conclude that $\lim _{k \rightarrow \infty}\left|d I C\left(M^{\prime}(k, 1)\right)\right|=\infty$. Since $\operatorname{dObj}\left(M^{\prime}(k, 1)\right)$ does not depend on $k$, it follows that $e\left(M^{\prime}(k, 1)\right)$ converges to zero as $k$ goes to infinity. This implies that if $k$ is large enough, then $e(\bar{M}(l, d))>e\left(M^{\prime}(k, 1)\right)$. By Lemma 6 , this contradicts the optimality of $M^{*}$.

\section{Continuity}

For all $(p, c) \in(1 / 2,1) \times \mathbb{R}_{+}$, let $M^{*}(p, c)$ denote an optimal ex-post efficient mechanism given the cost of information acquisition $c$ and the precision of the signals $p$. In what follows, we show that for a given number of voters $N$, if $\left\{\left(p_{n}, c_{n}\right)\right\}_{1}^{\infty}$ converges to $(p, c)$, then all converging subsequences of mechanisms $M^{*}\left(p_{n_{k}}, c_{n_{k}}\right)$ converge to an optimal mechanism $M^{*}(p, c)$. To make this claim precisely, one must define what it means for a sequence of mechanisms to converge. As we have already pointed out, a mechanism can be identified with the collection of the following probabilities:

$$
\{p(V(l, d), D, M) \mid D \in\{\operatorname{Maj}, \operatorname{Min}, C\}, l, d \in\{1, \ldots, N\},\} .
$$

Recall that $p(V(l, d), D, M)$ denotes the probability of making decision $D$ conditional on being at state $V(l, d)$. Hence for all $(l, d) \in \mathbb{N}$,

$$
\sum_{D \in\{M a j, M i n, C\}} p(V(l, d), D, M)=1 .
$$


Therefore, a mechanism can be defined as a point in $[0,1]^{3 N^{2}}$, which is a compact space.

Definition $4 M_{n}$ converges to $M$ if

$$
\max _{\substack{D \in\{M a j, M i n, C\} \\(l, d) \in N^{2}}}\left|p\left(V(l, d), D, M_{n}\right)-p(V(l, d), D, M)\right| \longrightarrow_{n \rightarrow \infty} 0 .
$$

We claim the following:

Proposition 4 Suppose that $\left\{\left(p_{n}, c_{n}\right)\right\}_{1}^{\infty}$ converges to $(p, c)$, and $M^{*}\left(p_{n}, c_{n}\right)(n \in \mathbb{N})$ are optimal mechanisms for $\left(p_{n}, c_{n}\right)$. Then there are subsequences $\left\{\left(p_{n_{k}}, c_{n_{k}}\right)\right\}_{1}^{\infty}$ of $\left\{\left(p_{n}, c_{n}\right)\right\}_{1}^{\infty}$ such that $\left\{M^{*}\left(p_{n_{k}}, c_{n_{k}}\right)\right\}_{1}^{\infty}$ is converging. Furthermore, the limit of any converging sequence of mechanisms $\left\{M^{*}\left(p_{n_{k}}, c_{n_{k}}\right)\right\}_{1}^{\infty}$ is optimal for $(p, c)$.

Proof. See Appendix A.

\section{Robustness}

A common critique of Bayesian mechanism design is that to design the optimal mechanism, the SP has to have perfect knowledge about the information structure of the environment. Hence, a natural question to ask is: What happens if the actual mechanism is not exactly the optimal one? In particular, we are interested in what happens if the mechanism is not even incentive compatible.

We show that if the mechanism is of the form described in Theorem 1, there always exists a unique symmetric mixed-strategy equilibrium. That is, if the mechanism can be characterized by a decreasing step function as in Theorem 1, even if it is not incentive compatible, there always exists a symmetric equilibrium in which the voters randomize between collecting information and reporting a signal randomly. If the decreasing step function is too large, then this equilibrium is one in which the voters do not collect any information. However, we show that if the mechanism is close to an incentive compatible mechanism, the probability that a voter makes a random report instead of collecting information is small. Hence, the value of the objective function of the SP is also close to the value corresponding to the incentive compatible mechanism.

Proposition 5 Let $M$ be a mechanism of the form described in Theorem 1. Then there exists a unique symmetric mixed-strategy equilibrium. In this equilibrium, the voters, conditional on being asked, collect information with probability $r(M)$ and make random reports with probability $(1-r(M))$.

Suppose $\left\{M_{n}\right\}_{1}^{\infty}$ converges to $M^{\prime}$ and all of these mechanisms have the form described in Theorem 1. Suppose that $M^{\prime}$ is incentive compatible. Then,

$$
\begin{aligned}
\lim _{n \rightarrow \infty} r\left(M_{n}\right) & =1, \\
\lim _{n \rightarrow \infty} \operatorname{Obj}\left(M_{n}\right) & =\operatorname{Obj}\left(M^{\prime}\right),
\end{aligned}
$$

where $\operatorname{Obj}(M)$ is the value of objective function of the $S P$ in $M$. 
Proof. See Appendix A.

In the proof we show that due to the particular form of the mechanism, there is strategic substitutability among the voters. That is, the less precise the signals of the other voters, the more incentive a voter has to collect information. Suppose that the voters randomize and collect information with probability $r$. Then the precision of the report is

$$
\widehat{p}(r)=p_{r}(\mathcal{H} \mid H)=p_{r}(\mathcal{L} \mid L)=r p+(1-r) \frac{1}{2} .
$$

(This is because the voters' randomization is independent of the realization of the signals.) Let $\widehat{d}(l, r)$ denote the difference between the numbers of different signals reported after asking $l$ voters. If $r_{1}>r_{2}$, then the distribution of $\widehat{d}\left(l, r_{1}\right)$ first-order stochastically dominates the distribution of $\widehat{d}\left(l, r_{2}\right)$. That is, after asking $l$ voters, the smaller $r$ is, the more evenly the reported signals are distributed. Therefore, as $r$ increases, the expected number of voters after which the SP makes a decision decreases. Hence, when a voter is asked to report a signal, the larger is $r$, the more likely that the voter is being asked after only a few other voters and the posterior of the SP is precise. These are exactly the events when the voter has little incentive to collect information. This shows that the smaller is $r$, the more incentive a voter has to acquire information. But strategic substitutability implies the uniqueness of a symmetric equilibrium. The second part of the proposition follows from continuity.

\subsection{Ex-ante Optimal Mechanism}

In this section, we show that the optimal mechanism sometimes involves ex-post inefficient decisions. That is, the mechanism characterized in Theorem 1 is not always optimal. This result is proved by contradiction. It will be shown that if the cost of information acquisition is small enough, then the optimal ex-post efficient mechanism can be improved upon by replacing a continuation mechanism with an ex-post inefficient continuation mechanism.

Let $p^{d}(V, D, M)$ denote the probability of making decision $D$ conditional on reaching $V$ and $M$, where $\operatorname{Maj}(\operatorname{Min})$ means taking action $a_{H}$ if and only if $d(V)+d>(<) 0$. Consider the following two mechanisms, $M_{1}$ and $M_{2}$ :

$$
\begin{aligned}
p^{1}\left(V(1,1), M a j, M_{1}\right) & =1 \\
p^{1}\left(V(1,-1), C, M_{1}\right) & =1 \\
p^{1}\left(V(2,0), M a j, M_{1}\right) & =1 \\
p^{1}\left(V(2,-2), M a j, M_{1}\right) & =1,
\end{aligned}
$$


and

$$
\begin{aligned}
p^{1}\left(V(1,1), M a j, M_{2}\right) & =1 \\
p^{1}\left(V(1,-1), C, M_{2}\right) & =1 \\
p^{1}\left(V(2,-2), C, M_{2}\right) & =p^{1}\left(V(2,0), C, M_{2}\right)=1 \\
p^{1}\left(V(3,1), M a j, M_{2}\right) & =p^{1}\left(V(3,-3), M a j, M_{2}\right)=1 \\
p^{1}\left(V(3,-1), C, M_{2}\right) & =1 \\
p^{1}\left(V(4,0), M i n, M_{2}\right) & =p^{1}\left(V(4,-2), \text { Min }, M_{2}\right)=1 .
\end{aligned}
$$

Employing $M_{1}$ at $V(K, 1)$ means the following. The SP asks an additional voter. If the voter confirms the posterior of the SP, he stops and makes the majority decision. If not, the SP asks an additional voter and makes the decision corresponding to the vote of the last voter. On the other hand, employing $M_{2}$ at $V(K, 1)$ means the following. At $V(K, 1)$, the SP asks an additional voter, and if she confirms his posterior, he makes the majority decision. If not, the SP asks two more voters. If their reports are the same, he again makes the majority decision. However, if the two voters report different signals, the SP asks an additional voter and makes the minority decision. In the following lemma we show that if $c$ is small enough, then $M_{2}$ is more efficient than $M_{1}$ at $V(K, 1)$.

Lemma 9 There exists a $\widetilde{c}(>0)$ such that whenever $c \in(0, \widetilde{c})$,

$$
e\left(M_{2}(K, 1)\right)>e\left(M_{1}(K, 1)\right) .
$$

Proof. See Appendix A.

We have to show that for some values of $c$, the optimal ex-post efficient mechanism indeed uses $M_{1}$ with probability between zero and one.

Lemma 10 For all $\widehat{c}(>0)$ there exist $N \in \mathbb{N}_{+}, c<\widehat{c}$ such that if there are $N$ voters and the cost of information acquisition is $c$, then for some $K \in \mathbb{N}, \operatorname{Pr}\left(M_{1}(K, 1) \mid M^{*}\right) \in(0,1)$.

Proof. See Appendix A.

Now we are ready to claim our third theorem.

Theorem 3 For all $\widehat{c}(>0)$ there exist $N \in \mathbb{N}_{+}, c<\widehat{c}$ such that if there are $N$ voters and the cost of information acquisition is $c$, then the ex-ante optimal mechanism involves ex-post inefficient decisions.

Proof. By the previous lemma, for all $\widehat{c}(>0)$ there exist $N \in \mathbb{N}_{+}, c<\widehat{c}$ such that if there are $N$ voters and the cost of information acquisition is $c$, then $\operatorname{Pr}\left(M_{1} \mid M^{*}\right) \in(0,1)$. In particular, this is true for $\widetilde{c}$ in the claim of Lemma 9. But if $c<\widetilde{c}$, then $e\left(M_{2}(K, 1)\right)>e\left(M_{1}(K, 1)\right)$ by Lemma 9. Hence, by Remark 3, the optimal ex-post efficient mechanism, $M^{*}$, cannot be optimal. Hence, the optimal mechanism involves ex-post inefficient decisions. 


\section{Discussion}

This paper analyzed optimal voting schemes in environments where information acquisition is costly and unobservable. We characterized the optimal ex-post efficient voting scheme (Theorem 1). We showed that the optimal mechanism can be essentially defined by a decreasing step function. For each number of voters already asked, this function assigns a value of the posterior. The Social Planner stops asking voters if and only if his posterior is more precise than the value corresponding to the number of voters already asked. Since the function is decreasing, this decision rule means that the more voters have already been asked, the less precise a posterior induces the Social Planner to act instead of acquiring more information. On top of the formal proof, we gave an informal explanation for this result (and justified our intuition by Propositions 2 and 3). On the one hand, the Social Planner wants to act only if his posterior is sufficiently precise. On the other hand, to give voters enough incentive to acquire information, he must make sure each voter is sufficiently likely to be decisive. A voter is more likely to be pivotal if the Social Planner's posterior is imprecise when the Social Planner makes a decision. Theorem 1 says that the Social Planner should make the voters pivotal along relatively long sequences. This is because a voter, conditional on being asked, assigns high probability to long sequences relative to the actual probability of those sequences. This intuition does not involve any assumption about the distribution of the states of the world and the signals. Hence, we conjecture that the statement of Theorem 1 is valid in one form or another under virtually any information structure.

What was essential in our computations is that the posterior of the SP was a function only of the number of reported signals and the difference between the numbers of different signals. It does not seem to be important that the posterior actually does not depend on the number of signals. This is because these two variables define the state space. Suppose, for example, that the range of signals is $\{\mathcal{H}, \mathcal{L}, \emptyset\}$ instead of $\{\mathcal{H}, \mathcal{L}\}$, and $p(\mathcal{H} \mid H)=p(\mathcal{L} \mid L)$ and $p(\emptyset \mid H)=p(\emptyset \mid L)$. That is, a voter may observe a signal $\emptyset$ and be unable to update her prior about the state of the world. The posterior of the SP remains a function only of the difference between the number of signals $\mathcal{H}$ and $\mathcal{L}$. We conjecture that the claim of Theorem 1 is still valid. The only difference is that all states of the form $V(l, d),|d| \leq l$, must be considered and not only those where $l-d$ is an even number.

Nonetheless, we view our model's assumptions about the information structure as restrictive. Only the particular assumed distributions enabled us to explicitly characterize the incentive compatibility constraint. Recall that having the explicit form of the incentive compatibility constraint made it possible to compute the efficiency of continuation mechanisms. These computations played a major role in the proof of Theorem 3, which states that if the cost of information acquisition is small enough, the ex-ante optimal voting mechanism sometimes necessarily involves ex-post inefficient decisions. The fact that ex-post inefficient decisions can increase ex-ante efficiency in voting models was also reported in Chwe (1999), Li (2001), and Gerardi and Yariv (2003). (We find this result surprising.) It says that the Social Planner can threaten the voters by committing to make ex-post inefficient decisions. This threat induces the voters to acquire information to avoid 
inefficient decisions. Other than this result, hardly anything is known about the ex-ante optimal scheme. Nonetheless, we view the problem of identifying optimal mechanisms that are ex-post inefficient as rather theoretical. We believe that in most economic and political situations of interest, commitment to inefficient actions is impossible.

A common critique of the mechanism described in this paper is that a voter cannot be absent. What happens if for some reason the SP must allow voters to be absent? We claim that the SP can achieve the same value of his objective function as without this restriction. Modify the mechanism described in Theorem 1 as follows. The possible actions of a voter are reporting signal $\mathcal{H}$ or $\mathcal{L}$ or being absent. If a voter is absent, the SP randomizes between signals $\mathcal{H}$ and $\mathcal{L}$ and continues to operate the mechanism as if the voter had reported the outcome of this randomization. From the voter's point of view, being absent is the same as reporting a signal randomly, given that the rest of the voters acquire information. Ex-post efficiency is not violated, either, since the SP makes a final decision only if the difference between the numbers of each type of signal is not zero. Hence the deviation of a single voter cannot make the final decision ex-post inefficient.

We assumed that the cost of acquiring information is the same for all voters. What happens if the voters' costs are heterogeneous? The derivation of the canonical mechanism (Lemma 2) is still valid. However, one cannot restrict attention to the uniform ordering of voters anymore. Clearly, the SP prefers to place the low-cost voters at the beginning of the sequences. On the other hand, a voter has little incentive to acquire information if she knows that she is at the beginning of a sequence. Hence, we conjecture that in the optimal mechanism, the low-cost voters are placed at the beginning of the ordering relatively more frequently but not surely. Also, the difficulty of analyzing that problem is that each voter who is ordered differently will have a different incentive compatibility constraint.

Finally, throughout the paper we maintained the assumption that utilities are not transferable. The Social Planner cannot use a transfer scheme to induce the voters to acquire information. If he could do so, he could implement the first-best mechanism. Such a mechanism would specify a transfer scheme that rewards a voter if her report matches the majority of other voters' reports and punishes the voter if her report differs from the majority. (For further details of such mechanisms, see Cremer and McLean (1988) and McAfee and Reny (1992).) We believe that many environments where small groups make decisions should be modeled with nontransferable utilities. 


\section{Appendix A}

Proof of Lemma 1. Using Bayes' rule

$$
p(H \mid s)=\frac{p(H \cap s)}{p(s)}=\frac{p(s \mid H) p(H)}{p(H) p(s \mid H)+p(L) p(s \mid L)} .
$$

Notice that

$$
p(s \mid H)=p^{\# \mathcal{H}} q^{\# \mathcal{L}}
$$

and

$$
p(s \mid L)=q^{\# \mathcal{H}} p^{\# \mathcal{L}} .
$$

Using the previous two formulas, and the assumption that $p(H)=p(L)=1 / 2$, (13) can be rewritten as

$$
p(H \mid s)=\frac{\frac{1}{2} p^{\# \mathcal{H}} q^{\# \mathcal{L}}}{\frac{1}{2}\left(p^{\# \mathcal{H}} q^{\# \mathcal{L}}+p^{\# \mathcal{L}} q^{\# \mathcal{H}}\right)}=\frac{p^{d}}{p^{d}+q^{d}} .
$$

Let $\mathcal{H} \cap s$ denote the set of events where after sequence $s$, signal $\mathcal{H}$ is realized. Then clearly

$$
\begin{aligned}
p(\mathcal{H} \cap s \mid H) & =p^{\# \mathcal{H}+1} q^{\# \mathcal{L}}, \\
p(\mathcal{H} \cap s \mid L) & =p^{\# \mathcal{L}} q^{\# \mathcal{H}+1} .
\end{aligned}
$$

Hence,

$$
\begin{aligned}
p(\mathcal{H} \mid s) & =\frac{p(\mathcal{H} \cap s)}{p(s)}=\frac{p(H) p(\mathcal{H} \cap s \mid H)+p(L) p(\mathcal{H} \cap s \mid L)}{p(H) p(s \mid H)+p(L) p(s \mid L)} \\
& =\frac{\frac{1}{2} p^{\# \mathcal{H}+1} q^{\# \mathcal{L}}+\frac{1}{2} p^{\# \mathcal{L}} q^{\# \mathcal{H}+1}}{\frac{1}{2} p^{\# \mathcal{H}} q^{\# \mathcal{L}}+\frac{1}{2} p^{\# \mathcal{L}} q^{\# \mathcal{H}}}=\frac{p^{d+1}+q^{d+1}}{p^{d}+q^{d}} .
\end{aligned}
$$

Proof of Lemma 3. Suppose, on the contrary, that (7) holds with strict inequality. Consider the following mechanism: employ the first-best mechanism with probability $\varepsilon(>0)$ and mechanism $M^{*}$ with probability $1-\varepsilon$. Clearly, this new mechanism increases the value of (1) compared with $M^{*}$. Furthermore, (10) is continuous in the probabilities $\rho(V, D, M)(D \in\{\operatorname{Maj}, \operatorname{Min}\})$, and these probabilities are continuous in $\varepsilon$. Hence, if $\varepsilon$ is small enough, the new mechanism is incentive compatible. This contradicts to the optimality of $M^{*}$.

Proof of Lemma 4. The statement of the lemma is true if the continuation mechanism specifies asking exactly one voter. This is because if $d=0$, then the expected posterior is $B(1)$, and the posterior at $V(l, d)$ is $1 / 2$. If $d \neq 0$, then from Lemma 1 it follows that

$$
B(|d|)=p(|d|) B(|d+1|)+q(|d|) B(|d-1|) .
$$

We proceed by induction with respect to the maximum number of voters asked in the continuation mechanism. Suppose that the claim of the lemma is satisfied whenever the maximum number of voters asked in a continuation mechanism is weakly less than $K$. Let $M(l, d)$ be a continuation 
mechanism in which the maximum number of voters asked is $K+1$. Let $M_{1}(l+1, d+1)$ and $M_{2}(l+1, d-1)$ be the continuation mechanisms induced by $M(l, d)$ at states $V(l+1, d+1)$ and $V(l+1, d-1)$, respectively. Notice that the maximum number of voters asked in $M_{1}(l+1, d+1)$ and $M_{2}(l+1, d-1)$ is at most $K$. Hence, by the inductive hypothesis,

$$
\begin{aligned}
& \sum_{V \in \mathcal{V}(N)} \rho^{d+1}\left(V, M a j, M_{1}(l+1, d+1)\right) B(|d(V)+d+1|) \geq B(|d+1|), \\
& \sum_{V \in \mathcal{V}(N)} \rho^{d-1}\left(V, M a j, M_{2}(l+1, d-1)\right) B(|d(V)+d-1|) \geq B(|d-1|) .
\end{aligned}
$$

Observe that

$$
\begin{aligned}
& \sum_{V \in \mathcal{V}(N)} \rho^{d}(V, M a j, M(l, d)) B(|d(V)+d|) \\
= & p(d) \sum_{V \in \mathcal{V}(N)} \rho^{d+1}\left(V, M a j, M_{1}(l+1, d+1)\right) B(|d(V)+d+1|) \\
& +q(d) \sum_{V \in \mathcal{V}(N)} \rho^{d-1}\left(V, M a j, M_{2}(l+1, d-1)\right) B(|d(V)+d-1|) \\
\geq & p(d) B(|d+1|)+q(d) B(|d-1|),
\end{aligned}
$$

where the inequality follows from (14). This is exactly the statement of the lemma for continuation mechanism $M$.

Proof of Lemma 7. Without loss of generality, assume that $d>0$. Assume by contradiction that

$$
\rho^{d}\left(V^{\prime}, M a j, M(l, d)\right)=0
$$

whenever $d\left(V^{\prime}\right)<0$. First, we show that

$$
\sum_{V \in \mathcal{V}(N)} \rho^{d}(V, M a j, M(l, d)) B(d(V)+d)(d(V)-(p-q) l(V))=0
$$

whenever (15) is satisfied. We prove it by induction on the maximum number of voters asked according to $M(l, d)$. If this number is one, then the previous expression is

$$
\begin{aligned}
& p(d) B(d+1)(1-(p-q))-q(d) B(d-1)(-1-(p-q)) \\
= & p(d) B(d+1)(2 q)+q(d) B(d-1)(2 p) .
\end{aligned}
$$

From Lemma 1,

$$
\begin{aligned}
& p(d) B(d+1) q=\frac{p^{d+1}+q^{d+1}}{p^{d}+q^{d}} \frac{p^{d+1}}{p^{d+1}+q^{d+1}} q \\
= & p q \frac{p^{d}}{p^{d}+q^{d}}
\end{aligned}
$$


and similarly

$$
\begin{aligned}
& q(d) B(d-1) p \\
= & \left(1-\frac{p^{d+1}+q^{d+1}}{p^{d}+q^{d}}\right) \frac{p^{d-1}}{p^{d-1}+q^{d-1}} p \\
= & p^{2} q \frac{p^{d-1}+q^{d-1}}{p^{d}+q^{d}} \frac{p^{d-1}}{p^{d-1}+q^{d-1}} \\
= & p q \frac{p^{d}}{p^{d}+q^{d}} .
\end{aligned}
$$

Hence (17) is zero. Suppose that the claim is true whenever the maximum number of voters asked is $K$, and mechanism $M(l, d)$ may ask $K+1$ voters. Let $M_{1}, M_{2}$ denote the continuation mechanisms generated by $M(l, d)$ at $V(l+1, d+1)$ and $V(l+1, d-1)$, respectively. If $M(l, d)$ satisfies (15), $M_{1}$ and $M_{2}$ also satisfy it. That is,

$$
\begin{aligned}
\rho^{d+1}\left(V^{\prime}, M a j, M_{1}(l+1, d+1)\right) & =0 \\
\rho^{d-1}\left(V^{\prime \prime}, M a j, M_{2}(l+1, d-1)\right) & =0
\end{aligned}
$$

whenever $d\left(V^{\prime}\right)+d+1<0$ and $d\left(V^{\prime \prime}\right)+d-1<0$. Since the maximum number of voters asked in $M(l, d)$ is $K+1$, the maximum number of voters asked in $M_{1}$ and $M_{2}$ is at most $K$. Therefore, by the inductive hypothesis,

$$
\begin{gathered}
\sum_{V \in \mathcal{V}(N)} \rho^{d+1}\left(V, M a j, M_{1}(l+1, d+1)\right) B(d(V)+d+1)(d(V)-(p-q) l(V))=0 \\
\sum_{V \in \mathcal{V}(N)} \rho^{d-1}\left(V, M a j, M_{2}(l+1, d-1)\right) B(d(V)+d-1)(-1-(p-q) l(V))=0 .
\end{gathered}
$$


Then

$$
\begin{aligned}
& \sum_{V \in \mathcal{V}(N)} \rho^{d}(V, M a j, M(l, d)) B(d(V)+d)(d(V)-(p-q) l(V)) \\
= & p(d) \sum_{V \in \mathcal{V}(N)} \rho^{d+1}\left(V, M a j, M_{1}(l+1, d+1)\right) B(d(V)+d+1)(d(V)+1-(p-q)(l(V)+1)) \\
& +q(d) \sum_{V \in \mathcal{V}(N)} \rho^{d-1}\left(V, M a j, M_{2}(l+1, d-1)\right) B(d(V)+d-1)(d(V)-1-(p-q)(l(V)+1)) \\
= & p(d) \sum_{V \in \mathcal{V}(N)} \rho^{d+1}\left(V, M a j, M_{1}(l+1, d+1)\right) B(d(V)+d+1)(d(V)-(p-q) l(V)) \\
& +p(d) \sum_{V \in \mathcal{V}(N)} \rho^{d+1}\left(V, M a j, M_{1}(l+1, d+1)\right) B(d(V)+d+1)(1-(p-q)) \\
& +q(d) \sum_{V \in \mathcal{V}(N)} \rho^{d-1}\left(V, M a j, M_{2}(l+1, d-1)\right) B(d(V)+d-1)(-1-(p-q) l(V)) \\
& q(d) \sum_{V \in \mathcal{V}(N)} \rho^{d-1}\left(V, M a j, M_{1}(l+1, d+1)\right) B(d(V)+d-1)(-1-(p-q)) \\
= & p(d) \sum_{V \in \mathcal{V}(N)} \rho^{d+1}\left(V, M a j, M_{1}(l+1, d+1)\right) B(d(V)+d+1)(1-(p-q)) \\
& +q(d) \sum_{V \in \mathcal{V}(N)} \rho^{d-1}\left(V, M a j, M_{2}(l+1, d-1)\right) B(d(V)+d-1)(-1-(p-q)) \\
= & p(d) B(d+1)(2 q)+q(d) B(d-1)(2 p)=0 .
\end{aligned}
$$

The third equality follows from (20) and the last one from (18) and (19).

We are ready to prove the lemma. First, notice that employing the continuation mechanism $M(l, d)$ instead of making the majority decision at $V(l, d)$ only decreases the value of the objective function of the SP by $c \bar{L}(M)$. This is because the expected posterior of the SP about the true state of the world, after deciding to employ $M(l, d)$ is the same as at $V(l, d)$, since he always takes the same action. That is,

$$
\sum_{V \in \mathcal{V}(N)} \rho^{d}(V, M a j, M(l, d)) B(d(V)+d)=B(d) .
$$

We show that it also destroys incentive compatibility. The change in incentive compatibility, 
$d I C(M(l, d))$ is

$$
\begin{aligned}
& \frac{p-q}{4 p q} \sum_{V \in \mathcal{V}(N)} \rho^{d}(V, M a j, M(l, d)) B(d(V)+d)[d(V)+d-(p-q)(l(V)+l)] \\
& -c \bar{L}(M(l, d))-\frac{p-q}{4 p q} B(d)[d-(p-q) l] \\
= & \frac{p-q}{4 p q}\left[\sum_{V \in \mathcal{V}(N)} \rho^{d}(V, M a j, M(l, d)) B(d(V)+d)-B(d)\right][d-(p-q) l] \\
& \frac{p-q}{4 p q}\left[\sum_{V \in \mathcal{V}(N)} \rho^{d}(V, M a j, M(l, d)) B(d(V)+d)(d(V)-(p-q) l(V))\right]-c \bar{L}(M(l, d)) \\
= & -c \bar{L}(M(l, d)) .
\end{aligned}
$$

The term in square brackets in the third line is zero by (21). Also, the term in square brackets in the fourth line is zero by (16). This means employing the continuation mechanism $M$ at $V(l, d)$ decreases the value of the objective function as well as destroying incentive compatibility, contradicting the optimality of the mechanism $M^{*}$.

Proof of Lemma 8. Suppose that $M^{*}$ is an optimal mechanism among the ex-post efficient ones and that it involves randomization at states $V(l, d)$ and $V\left(l^{\prime}, d^{\prime}\right)$ and $(l,|d|) \neq\left(l^{\prime},\left|d^{\prime}\right|\right)$. From Lemma 6 , it follows that $e(M(l, d))=e\left(M\left(l^{\prime}, d^{\prime}\right)\right)$, and therefore

$$
\frac{d \operatorname{Obj}(M(l, d))}{d \operatorname{Obj}\left(M\left(l^{\prime}, d^{\prime}\right)\right)} d I C\left(M\left(l^{\prime}, d^{\prime}\right)\right)=d I C(M(l, d)) .
$$

We consider two cases. In Case 1,

$$
\operatorname{Pr}\left(M(l, d) \mid M^{*}\right) \frac{p\left(V(l, d), M^{*}\right) d \operatorname{Obj}(M(l, d))}{p\left(V\left(l^{\prime}, d^{\prime}\right), M^{*}\right) d \operatorname{Obj}\left(M\left(l^{\prime}, d^{\prime}\right)\right)}<1-\operatorname{Pr}\left(M\left(l^{\prime}, d^{\prime}\right) \mid M^{*}\right),
$$

while in Case 2, the opposite inequality holds. In both of these cases we construct an optimal mechanism that randomizes at strictly fewer states than $M^{*}$.

Case 1. Consider the following new mechanism. The new mechanism differs from $M^{*}$ only at states $V(l, d)$ and $V\left(l^{\prime}, d^{\prime}\right)$. Conditional on being at $V(l, d)$, it stops and makes the majority decision. Conditional on being at $V\left(l^{\prime}, d^{\prime}\right)$, it employs $M\left(l^{\prime}, d^{\prime}\right)$ with probability

$$
\operatorname{Pr}\left(M\left(l^{\prime}, d^{\prime}\right) \mid M^{*}\right)+\operatorname{Pr}\left(M(l, d) \mid M^{*}\right) \frac{p\left(V(l, d), M^{*}\right) d \operatorname{Obj}(M(l, d))}{p\left(V\left(l^{\prime}, d^{\prime}\right), M^{*}\right) d \operatorname{Obj}\left(M\left(l^{\prime}, d^{\prime}\right)\right)} .
$$

The change in the objective function is

$$
\begin{aligned}
& p\left(V\left(l^{\prime}, d^{\prime}\right), M^{*}\right) \frac{p\left(V(l, d), M^{*}\right) \operatorname{Pr}\left(M(l, d) \mid M^{*}\right) d \operatorname{Obj}(M(l, d))}{p\left(V\left(l^{\prime}, d^{\prime}\right), M^{*}\right) d \operatorname{Obj}\left(M\left(l^{\prime}, d^{\prime}\right)\right)} d \operatorname{Obj}\left(M\left(l^{\prime}, d^{\prime}\right)\right) \\
& -p\left(V(l, d), M^{*}\right) \operatorname{Pr}\left(M(l, d) \mid M^{*}\right) d \operatorname{Obj}(M(l, d)) \\
= & 0 .
\end{aligned}
$$


The change in the incentive compatibility constraint is

$$
\begin{aligned}
& p\left(V\left(l^{\prime}, d^{\prime}\right), M^{*}\right) \frac{p\left(V(l, d), M^{*}\right) \operatorname{Pr}\left(M(l, d) \mid M^{*}\right) d \operatorname{Obj}(M(l, d))}{p\left(V\left(l^{\prime}, d^{\prime}\right), M^{*}\right) d \operatorname{Obj}\left(M\left(l^{\prime}, d^{\prime}\right)\right)} d I C\left(M\left(l^{\prime}, d^{\prime}\right)\right) \\
& -p\left(V(l, d), M^{*}\right) \operatorname{Pr}\left(M(l, d) \mid M^{*}\right) d I C(M(l, d)) \\
= & p\left(V\left(l^{\prime}, d^{\prime}\right), M^{*}\right) \frac{p\left(V(l, d), M^{*}\right) \operatorname{Pr}\left(M(l, d) \mid M^{*}\right)}{p\left(V\left(l^{\prime}, d^{\prime}\right), M^{*}\right)} d I C(M(l, d)) \\
& -p\left(V(l, d), M^{*}\right) \operatorname{Pr}\left(M(l, d) \mid M^{*}\right) d I C(M(l, d)) \\
= & 0 .
\end{aligned}
$$

The first inequality follows from (22). Since $M^{*}$ was optimal and incentive compatible, the new mechanism is also optimal and incentive compatible.

Case 2. Again, the new mechanism differs from $M^{*}$ only at states $V(l, d)$ and $V\left(l^{\prime}, d^{\prime}\right)$. Conditional on being at $V(l, d)$, it employs $M(l, d)$ with probability

$$
\operatorname{Pr}\left(M(l, d) \mid M^{*}\right)-\frac{p\left(V\left(l^{\prime}, d^{\prime}\right), M^{*}\right)\left(1-\operatorname{Pr}\left(M\left(l^{\prime}, d^{\prime}\right) \mid M^{*}\right)\right) d \operatorname{Obj}\left(M\left(l^{\prime}, d^{\prime}\right)\right)}{p\left(V(l, d), M^{*}\right) d \operatorname{Obj}(M(l, d))} .
$$

Recall that in Case 2,

$$
\operatorname{Pr}\left(M(l, d) \mid M^{*}\right) \frac{p\left(V(l, d), M^{*}\right) d \operatorname{Obj}(M(l, d))}{p\left(V\left(l^{\prime}, d^{\prime}\right), M^{*}\right) d \operatorname{Obj}\left(M\left(l^{\prime}, d^{\prime}\right)\right)} \geq 1-\operatorname{Pr}\left(M\left(l^{\prime}, d^{\prime}\right) \mid M^{*}\right),
$$

so the previous expression is weakly positive. Conditional on being at $V\left(l^{\prime}, d^{\prime}\right)$, the new mechanism employs $M\left(l^{\prime}, d^{\prime}\right)$ with probability one. The change in the objective function is

$$
\begin{aligned}
& p\left(V\left(l^{\prime}, d^{\prime}\right), M^{*}\right)\left(1-\operatorname{Pr}\left(M\left(l^{\prime}, d^{\prime}\right) \mid M^{*}\right)\right) d \operatorname{Obj}\left(M\left(l^{\prime}, d^{\prime}\right)\right) \\
& -p\left(V(l, d), M^{*}\right) \frac{p\left(V\left(l^{\prime}, d^{\prime}\right), M^{*}\right)\left(1-\operatorname{Pr}\left(M\left(l^{\prime}, d^{\prime}\right) \mid M^{*}\right)\right) d \operatorname{OObj}\left(M\left(l^{\prime}, d^{\prime}\right)\right)}{p\left(V(l, d), M^{*}\right) d \operatorname{Obj}(M(l, d))} d \operatorname{Obj}(M(l, d)) \\
= & 0 .
\end{aligned}
$$

The change in the incentive compatibility constraint is

$$
\begin{aligned}
& p\left(V\left(l^{\prime}, d^{\prime}\right), M^{*}\right)\left(1-\operatorname{Pr}\left(M\left(l^{\prime}, d^{\prime}\right) \mid M^{*}\right)\right) d I C\left(M\left(l^{\prime}, d^{\prime}\right)\right) \\
& -p\left(V(l, d), M^{*}\right) \frac{p\left(V\left(l^{\prime}, d^{\prime}\right), M^{*}\right)\left(1-\operatorname{Pr}\left(M\left(l^{\prime}, d^{\prime}\right) \mid M^{*}\right)\right) d \operatorname{Obj}\left(M\left(l^{\prime}, d^{\prime}\right)\right)}{p\left(V(l, d), M^{*}\right) d \operatorname{Obj}(M(l, d))} d I C(M(l, d)) \\
= & 0 .
\end{aligned}
$$

Again, since $M^{*}$ was optimal and incentive compatible, the new mechanism is also optimal and incentive compatible.

Therefore, whenever an optimal mechanism involves randomization at two different states, one can always find a different optimal mechanism that randomizes at strictly fewer states. From this, the first part of the statement of the lemma follows.

It remains to show that if the first-best mechanism is not incentive compatible, $M^{*}$ generically involves randomization. However, there are only finitely many deterministic mechanisms. Hence, 
the left-hand side of the incentive compatibility constraint (10) can take only finitely many values given $p$. From Lemma 3, it follows that (10) must hold with inequality. This means that for any $p$, there are only finitely many values of $c$ for which (10) holds with equality.

Proof of Theorem 2. We have shown in Lemma 8 that the optimal mechanisms generically involve randomization. We claim that if there are at least two optimal mechanisms, then there must exist two continuation mechanisms that have the same efficiency. If the two mechanisms are characterized by the same function, $f$, described in Theorem 1, and they differ only in the randomization, then this follows immediately from Lemma 6 . If the two mechanisms differ in the function $f$, then take the probability mixture of the two. By Remark 2, this mixture is also an optimal mechanism and involves randomization in at least two places.

By Lemma 6, it is enough to show that, generically, two deterministic continuation mechanisms that may be used in the optimal mechanism have different efficiencies. Let us introduce two new pieces of notation:

$$
\begin{aligned}
& \Psi(M(l, d)) \\
= & N \sum_{V \in \mathcal{V}(N)} \rho^{d}(V, M a j, M(l, d)) B(|d(V)+d|) \\
& +N \sum_{V \in \mathcal{V}(N)} \rho^{d}(V, M i n, M(l, d)) R(|d(V)+d|) \\
& -N B(|d|), \\
& \Gamma(M(l, d)) \\
= & \frac{p-q}{4 p q} \sum_{V \in \mathcal{V}(N)} \rho^{d}(V, M a j, M(l, d)) B(|d(V)+d|)[|d(V)+d|-(p-q)(l(V)+l)] \\
& +\frac{p-q}{4 p q} \sum_{V \in \mathcal{V}(N)} \rho^{d}(V, M i n, M(l, d)) R(|d(V)+d|)[-|d(V)+d|-(p-q)(l(V)+l)] \\
& -\frac{p-q}{4 p q} B(|d|)[|d|-(p-q) l] .
\end{aligned}
$$

Then the efficiency of $M$ can be written as

$$
e(M(l, d))=\left|\frac{\Psi(M(l, d))-c \bar{L}_{d}(M)}{\Gamma(M(l, d))-c \bar{L}_{d}(M)}\right| .
$$


Notice that $\Psi$ and $\Gamma$ do not depend on $c$, hence

$$
\begin{aligned}
& \left|\frac{d e(M(l, d))}{d c}\right| \\
= & \left|\frac{-\bar{L}_{d}(M)\left(\Gamma(M(l, d))-c \bar{L}_{d}(M)\right)+\bar{L}_{d}(M)\left(\Psi(M(l, d))-c \bar{L}_{d}(M)\right)}{\left(\Gamma(M(l, d))-c \bar{L}_{d}(M)\right)^{2}}\right| \\
= & \left|\frac{-\bar{L}_{d}(M)}{\Psi(M(l, d))-c \bar{L}_{d}(M)} \frac{\Psi(M(l, d))-c \bar{L}_{d}(M)}{\Gamma(M(l, d))-c \bar{L}_{d}(M)}+\frac{\bar{L}_{d}(M)}{\Psi(M(l, d))-c \bar{L}_{d}(M)} \frac{\left(\Psi(M(l, d))-c \bar{L}_{d}(M)\right)^{2}}{\left(\Gamma(M(l, d))-c \bar{L}_{d}(M)\right)^{2}}\right| \\
= & \left|\frac{\bar{L}_{d}(M)}{\Psi(M(l, d))-c \bar{L}_{d}(M)}\left(\frac{\left(\Psi(M(l, d))-c \bar{L}_{d}(M)\right)^{2}}{\left(\Gamma(M(l, d))-c \bar{L}_{d}(M)\right)^{2}}-\frac{\Psi(M(l, d))-c \bar{L}_{d}(M)}{\Gamma(M(l, d))-c \bar{L}_{d}(M)}\right)\right| \\
= & \left|\frac{\bar{L}_{d}(M)}{\Psi(M(l, d))-c \bar{L}_{d}(M)}\left(e^{2}(M(l, d))+e(M(l, d))\right)\right| .
\end{aligned}
$$

Let us now assume that the efficiency of $M_{1}$ at $V\left(l_{1}, d_{1}\right)$ is the same as the efficiency of $M_{2}$ at $V\left(l_{2}, d_{2}\right)$ for a positive measure of $(p, c) \in \mathbb{R}^{2}$, that is, $e\left(M_{1}\left(l_{1}, d_{1}\right)\right)=e\left(M_{2}\left(l_{2}, d_{2}\right)\right)$. Without loss of generality, assume that $d_{1}, d_{2}>0$. (This can be done because the optimal mechanism can be assumed to be symmetric with respect to $\mathcal{H}$ and $\mathcal{L}$.) This means, in particular, that for a positive measure of $(p, c) \in \mathbb{R}^{2}$

$$
\left|\frac{d e\left(M_{1}\left(l_{1}, d_{1}\right)\right)}{d c}\right|=\left|\frac{d e\left(M_{2}\left(l_{2}, d_{2}\right)\right)}{d c}\right|,
$$

and from (23),

$$
\left|\frac{\bar{L}_{d_{1}}\left(M_{1}\right)}{\Psi\left(M_{1}\left(l_{1}, d_{1}\right)\right)-c \bar{L}_{d_{1}}\left(M_{1}\right)}\right|=\left|\frac{\bar{L}_{d_{2}}\left(M_{2}\right)}{\Psi\left(M_{2}\left(l_{2}, d_{2}\right)\right)-c \bar{L}_{d_{2}}\left(M_{2}\right)}\right|
$$

and therefore

$$
\left|\frac{\Psi\left(M_{1}\left(l_{1}, d_{1}\right)\right)}{\bar{L}_{d_{1}}\left(M_{1}\right)}\right|=\left|\frac{\Psi\left(M_{2}\left(l_{2}, d_{2}\right)\right)}{\bar{L}_{d_{2}}\left(M_{2}\right)}\right|
$$

for a positive measure of $p^{\prime} s$. We will show that this is impossible for the continuation mechanisms that are potentially used in an optimal ex-post efficient mechanism. We show that both sides of the previous equality must be a rational fraction function. That is, both the numerator and the denominator are polynomials. Suppose that $M_{1}$ and $M_{2}$ are feasible continuation mechanisms in the optimal one. Hence, if $l_{1}<l_{2}$, then $d_{1}>d_{2}$ by Theorem 1 .

Next, we analyze the functional form of $\Psi(M(l, d)) / \bar{L}_{d}(M)$ :

$$
\Psi(M(l, d))=N\left[\sum_{V\left(l^{\prime}, d^{\prime}\right) \in \mathcal{V}^{*}(N)} n\left(l^{\prime}, d^{\prime}\right) p\left(l^{\prime}, d^{\prime}\right) B\left(\left|d^{\prime}\right|\right)-B(|d|)\right],
$$

where $\mathcal{V}^{*}(N)$ denotes the set of states in which the majority decision is made; $n\left(l^{\prime}, d^{\prime}\right)$ denotes the cardinality of the set of sequences that lead to $V\left(l^{\prime}, d^{\prime}\right)$ from $V(l, d)$ according to the optimal mechanism; and $p\left(l^{\prime}, d^{\prime}\right)$ denotes the probability of such a sequence conditional on being at state 
$V(l, d) . p\left(l^{\prime}, d^{\prime}\right)$ depends only on the realization of the signals, not on the mechanism. From the proof of Theorem 1 , it is clear that $n(l+1, d+1)=1$. That is, if in the continuation mechanism the first voter confirms the posterior, the mechanism stops and the majority decision is made. Furthermore,

$$
p(l+1, d+1) B(d+1)=\frac{p^{d+1}}{p^{d}+q^{d}} .
$$

Also notice that if the first voter does not confirm the posterior of the SP, any sequence realized in the continuation mechanism must contain weakly more signals $\mathcal{L}$ than signals $\mathcal{H}$. Suppose that $s$ contains $i$ signals $\mathcal{H}$ and $i+d^{\prime}$ signals $\mathcal{L}$ (where $d^{\prime} \geq 0$ ). Next, we compute the probability of $s$ conditional on being at $V(l, d)$ :

$$
p(s \mid d)=\frac{p^{i} q^{i}\left(p^{d^{\prime}} q^{d}+p^{d} q^{d^{\prime}}\right)}{p^{d}+q^{d}} .
$$

From Lemma 1,

$$
B\left(\left|d-d^{\prime}\right|\right)=\frac{p^{\left|d-d^{\prime}\right|}}{p^{\left|d-d^{\prime}\right|}+q^{\left|d-d^{\prime}\right|}} .
$$

Hence, if $d \geq d^{\prime}$,

$$
\begin{aligned}
p(s \mid d) B\left(\left|d-d^{\prime}\right|\right) & =p\left(l+2 i+d^{\prime}, d+d^{\prime}\right) B\left(\left|d-d^{\prime}\right|\right) \\
& =\frac{p^{i} q^{i}(p q)^{d^{\prime}}\left(q^{d-d^{\prime}}+p^{d-d^{\prime}}\right)}{p^{d}+q^{d}} \frac{p^{d-d^{\prime}}}{p^{d-d^{\prime}}+q^{d-d^{\prime}}}=\frac{p^{i+d} q^{i+d^{\prime}}}{p^{d}+q^{d}},
\end{aligned}
$$

and if $d<d^{\prime}$,

$$
\begin{aligned}
p(s \mid d) B\left(\left|d+d^{\prime}\right|\right) & =p\left(l+2 i+d^{\prime}, d+d^{\prime}\right) B\left(\left|d+d^{\prime}\right|\right) \\
& =\frac{p^{i} q^{i}(p q)^{d}\left(q^{d^{\prime}-d}+p^{d^{\prime}-d}\right)}{p^{d}+q^{d}} \frac{p^{d^{\prime}-d}}{p^{d^{\prime}-d}+q^{d^{\prime}-d}}=\frac{p^{i+d^{\prime}} q^{i+d}}{p^{d}+q^{d}} .
\end{aligned}
$$

Recall that $q=1-p$, hence both $p^{i+d} q^{i+d^{\prime}}$ and $p^{i+d^{\prime}} q^{i+d}$ are polynomials. The degrees of these polynomials are

$$
2 i+d^{\prime}+d=l(s)+d .
$$

Now we turn to the functional form of $\bar{L}_{d}(M)$ :

$$
\bar{L}_{d}(M)=\sum_{V\left(l^{\prime}, d^{\prime}\right) \in \mathcal{V}^{*}(N)} n\left(l^{\prime}, d^{\prime}\right) p\left(l^{\prime}, d^{\prime}\right)\left(l^{\prime}-l\right) .
$$

Again,

$$
p(s \mid d) l(s)=p\left(l+2 i+d^{\prime}, d+d^{\prime}\right)\left(2 i+d^{\prime}\right)=\frac{p^{i} q^{i}\left(p^{d^{\prime}} q^{d}+p^{d} q^{d^{\prime}}\right)}{p^{d}+q^{d}} l(s) .
$$

The numerator of the previous expression is a polynomial. The degree of this polynomial is again

$$
2 i+d^{\prime}+d=l(s)+d .
$$


Each term in both $\bar{L}_{d}(M)$ and $\Psi(M(l, d))$ is divided by $p^{d}+q^{d}$. Hence those terms cancel out from $\Psi(M(l, d)) / \bar{L}_{d}(M)$, and what remains is a rational fraction function. If two rational fraction functions agree on a positive measure of $p^{\prime} s$, they must be identical. That is, (25) must be an identity. In particular, the ratio of the coefficients of the terms with the largest degree in the numerator and in the dominator must be the same. From (26) and (27) it follows that the absolute value of the ratio of the coefficients corresponding to the terms with the largest power is $1 / l(s)$. Where $s$ is a longest possible sequence reaching a state where the majority decision is made from $V(l, d)$. Let $K$ denote the largest number of voters potentially asked in the optimal mechanism. Then, from Theorem 1 , there are sequences from both $V\left(l_{1}, d_{1}\right)$ and $V\left(l_{2}, d_{2}\right)$ that reach $V(K, 1)$ according to the optimal mechanism. Furthermore, these are the longest sequences in the continuation mechanisms. However, the longest possible such a sequence has length $K-l_{1}$ in $M_{1}\left(l_{1}, d_{1}\right)$ and $K-l_{2}$ in $M_{2}\left(l_{2}, d_{2}\right)$, so it is different for different continuation mechanisms.

Proof of Proposition 4. Since $M^{*}\left(p_{n}, c_{n}\right) \in[0,1]^{3 N^{2}}$, which is a compact space, there must be a subsequence $\left\{\left(p_{n_{k}}, c_{n_{k}}\right)\right\}_{1}^{\infty}$ such that $M^{*}\left(p_{n_{k}}, c_{n_{k}}\right)$ converges to a mechanism $M$. We have to show that $M$ is optimal for $(p, c)$. That is, we have to show that $M$ is incentive compatible and maximizes the value of the SP's objective function.

Suppose that $M$ is not incentive compatible for $(p, c)$. This means there exists an $\varepsilon(>0)$ such that

$$
\begin{aligned}
& \frac{p-q}{4 p q} \sum_{V \in \mathcal{V}(N)} \rho(V, M a j, M) B(|d(V)|)[|d(V)|-(p-q) l(V)] \\
& +\frac{p-q}{4 p q} \sum_{V \in \mathcal{V}(N)} \rho(V, M a j, M) R(|d(V)|)[-|d(V)|-(p-q) l(V)] \\
< & c \bar{L}(M)+\varepsilon .
\end{aligned}
$$

However, both sides of (10) are continuous in $p, c$ as well as in $\rho(V, D, M)^{\prime} s$. Hence there exists a $\delta(>0)$ such that if

$$
\left|p^{\prime}-p\right|,\left|c^{\prime}-c\right|,\left|\rho(V, D, M)-\rho\left(V, D, M^{\prime}\right)\right|<\delta \text { for all } V \in \mathcal{V}(N), D \in\{\text { Maj, Min }, C\},
$$

then

$$
\begin{aligned}
& \frac{p^{\prime}-q^{\prime}}{4 p^{\prime} q^{\prime}} \sum_{V \in \mathcal{V}(N)} \rho\left(V, M a j, M^{\prime}\right) B(|d(V)|)\left[|d(V)|-\left(p^{\prime}-q^{\prime}\right) l(V)\right] \\
& +\frac{p^{\prime}-q^{\prime}}{4 p^{\prime} q^{\prime}} \sum_{V \in \mathcal{V}(N)} \rho\left(V, M a j, M^{\prime}\right) R(|d(V)|)\left[-|d(V)|-\left(p^{\prime}-q^{\prime}\right) l(V)\right] \\
< & c^{\prime} \bar{L}\left(M^{\prime}\right) .
\end{aligned}
$$

That is, $M^{\prime}$ is not incentive compatible. Since $\left\{\left(p_{n_{k}}, c_{n_{k}}\right)\right\}_{1}^{\infty}$ converges to $(p, c)$ and $M^{*}\left(p_{n_{k}}, c_{n_{k}}\right)$ converges to $M$, there exists an $n_{0} \in \mathbb{N}$ such that if $n_{k}>n_{0}, p^{\prime}=p_{n_{k}}, c^{\prime}=c_{n_{k}}$ and $M^{\prime}=$ $M^{*}\left(p_{n_{k}}, c_{n_{k}}\right)$, then (28) is satisfied. This would imply that $M^{*}\left(p_{n_{k}}, c_{n_{k}}\right)$ is not incentive compatible, which is a contradiction. 
It remains to show that $M$ is indeed optimal for $(p, c)$. Denote the value of the objective function corresponding to a mechanism $M^{\prime}$ and $\left(p^{\prime}, c^{\prime}\right)$ by $O b j_{p^{\prime}, c^{\prime}}\left(M^{\prime}\right)$. First, notice that the objective function of the SP is also continuous in $p, c$ as well as in $\rho(V, D, M)^{\prime} s$. Hence, $O b j_{p_{n_{k}}, c_{n_{k}}}\left(M^{*}\left(p_{n_{k}}, c_{n_{k}}\right)\right)$ converges to $O b j_{p, c}(M)$. Suppose, by contradiction, that $M$ is not optimal for $(p, c)$. Then there exists an incentive compatible mechanism $M^{\prime}$ such that

$$
O b j_{p, c}\left(M^{\prime}\right)-O b j_{p, c}(M)=\delta>0
$$

As $\left\{\left(p_{n_{k}}, c_{n_{k}}\right)\right\}_{1}^{\infty}$ converges to $(p, c)$,

$$
\begin{aligned}
& \frac{p_{n_{k}}-q_{n_{k}}}{4 p_{n_{k}} q_{n_{k}}} \sum_{V \in \mathcal{V}(N)} \rho\left(V, M a j, M^{\prime}\right) B(|d(V)|)\left[|d(V)|-\left(p_{n_{k}}-q_{n_{k}}\right) l(V)\right] \\
& +\frac{p_{n_{k}}-q_{n_{k}}}{4 p_{n_{k}} q_{n_{k}}} \sum_{V \in \mathcal{V}(N)} \rho\left(V, M a j, M^{\prime}\right) R(|d(V)|)\left[-|d(V)|-\left(p_{n_{k}}-q_{n_{k}}\right) l(V)\right] \\
\longrightarrow & { }_{n_{k} \rightarrow \infty} c_{n_{k}} \bar{L}(M) .
\end{aligned}
$$

For each $\left(p_{n_{k}}, c_{n_{k}}\right)$, let us define a mechanism $M\left(p_{n_{k}}, c_{n_{k}}\right)$ as follows. With probability $\left(1-\varepsilon_{n_{k}}\right)$, the mechanism is $M^{\prime}$ and with probability $\varepsilon_{n_{k}}$ the SP asks only one voter to acquire information and decides according to her report. Furthermore, choose $\varepsilon_{n_{k}}$ such that the incentive compatibility constraint, (10), is satisfied with equality. From (29), it follows that as $n_{k}$ goes to infinity, $\varepsilon_{n_{k}}$ converges to zero. Hence,

$$
\lim _{n_{k} \rightarrow \infty} O b j_{p_{n_{k}}, c_{n_{k}}}\left(M\left(p_{n_{k}}, c_{n_{k}}\right)\right)=O b j_{p, c}\left(M^{\prime}\right) .
$$

This contradicts the optimality of the sequence $M^{*}\left(p_{n_{k}}, c_{n_{k}}\right)$, since from

$$
\lim _{n_{k} \rightarrow \infty} \operatorname{Obj}\left(M^{*}\left(p_{n_{k}}, c_{n_{k}}\right)\right)=\operatorname{Obj}(M)<\operatorname{Obj}\left(M^{\prime}\right)=\lim _{n_{k} \rightarrow \infty} \operatorname{Obj}\left(M\left(p_{n_{k}}, c_{n_{k}}\right)\right)
$$

it follows that if $n_{k}$ is large enough,

$$
\operatorname{Obj}\left(M^{*}\left(p_{n_{k}}, c_{n_{k}}\right)\right)<\operatorname{Obj}\left(M\left(p_{n_{k}}, c_{n_{k}}\right)\right) .
$$

Before we prove Proposition 5, we prove two lemmas.

Lemma 11 Let $a(., \widehat{p})$ be a measure on $\{1, \ldots, N\}$, that is, $\sum_{l=1}^{N} a(l, \widehat{p})=1$ and $a(l, \widehat{p}) \geq 0$. Further assume that if $\widehat{p}>\widehat{p}^{\prime}$, then a $(l, \widehat{p})$ first-order stochastically dominates a $(l, \widehat{p})$. Assume that $b(l, \widehat{p})$ is increasing in $l$ and decreasing in $\widehat{p}$. Then

$$
\sum_{l \in \mathbb{N}} a(l, \widehat{p}) b(l, \widehat{p})
$$

is decreasing in $\widehat{p}$. 
Proof. Suppose that $\widehat{p}>\widehat{p}^{\prime}$. Then

$$
\begin{aligned}
& \sum_{l \in \mathbb{N}} a(l, \widehat{p}) b(l, \widehat{p})-\sum_{l \in \mathbb{N}} a\left(l, \widehat{p}^{\prime}\right) b\left(l, \widehat{p}^{\prime}\right) \\
= & \sum_{l \in \mathbb{N}}\left(a(l, \widehat{p})-a\left(l, \widehat{p}^{\prime}\right)\right) b(l, \widehat{p}) \\
& +\sum_{l \in \mathbb{N}} a\left(l, \widehat{p}^{\prime}\right)\left(b(l, \widehat{p})-b\left(l, \widehat{p}^{\prime}\right)\right) .
\end{aligned}
$$

Since $a(., \widehat{p})$ first-order stochastically dominates $a\left(., \widehat{p}^{\prime}\right)$ and $b(l, \widehat{p})$ is increasing in $l$, the first term is (weakly) negative. Since $b(l, \widehat{p})$ is decreasing in $\widehat{p}$, the second term is also negative.

Lemma 12 Let $f$ and $g$ be probability distributions over $\{1, \ldots, N\}$. Suppose that

$$
\frac{g(i)}{g(i+1)} \geq \frac{f(i)}{f(i+1)} \text { for all } i \in\{1, \ldots, N-1\} .
$$

Then $g$ first-order stochastically dominates $f$.

Proof. Let $a_{i+1}$ denote $g(i+1) / g(i)$ and let $b_{i+1}$ denote $f(i+1) / f(i)$ for all $i \in\{1, \ldots, N-1\}$. Let $a_{1}=b_{1}=1$. By the hypothesis of the lemma, $a_{i} \leq b_{i}$ for all $i \in\{1, \ldots, N-1\}$. Then $g(i)$ can be written as $g(1) \prod_{j=1}^{i} a_{j}$, and similarly, $f(i)=f(1) \prod_{j=1}^{i} b_{j}$. To show that $g$ first-order stochastically dominates $f$, we have to prove that

$$
\sum_{i=1}^{j} g(i) \geq \sum_{i=1}^{j} f(i) \text { for all } j \in\{1, \ldots, N\} .
$$

Since $g$ and $f$ are probability distributions,

$$
\begin{aligned}
& g(1) \sum_{i=1}^{N} \prod_{j=1}^{i} a_{j}=1, \\
& f(1) \sum_{i=1}^{N} \prod_{j=1}^{i} b_{j}=1 .
\end{aligned}
$$

Therefore,

$$
g(1)=\frac{1}{\sum_{i=1}^{N} \prod_{j=1}^{i} a_{j}} \geq \frac{1}{\sum_{i=1}^{N} \prod_{j=1}^{i} b_{j}}=f(1),
$$

where the inequality follows from $a_{i} \leq b_{i}$. Then

$$
\begin{aligned}
& \sum_{i=1}^{K} g(i)=g(1) \sum_{i=1}^{K} \prod_{j=1}^{i} a_{j}=\frac{\sum_{i=1}^{K} \prod_{j=1}^{i} a_{j}}{\sum_{i=1}^{N} \prod_{j=1}^{i} a_{j}}, \\
& \sum_{i=1}^{K} f(i)=f(1) \sum_{i=1}^{K} \prod_{j=1}^{i} b_{j}=\frac{\sum_{i=1}^{K} \prod_{j=1}^{i} b_{j}}{\sum_{i=1}^{N} \prod_{j=1}^{i} b_{j}} .
\end{aligned}
$$

We have to show that

$$
\left(\sum_{i=1}^{K} \prod_{j=1}^{i} a_{j}\right)\left(\sum_{i=1}^{N} \prod_{j=1}^{i} b_{j}\right) \geq\left(\sum_{i=1}^{N} \prod_{j=1}^{i} a_{j}\right)\left(\sum_{i=1}^{K} \prod_{j=1}^{i} b_{j}\right) .
$$


The previous inequality can be rewritten as

$$
\left(\sum_{i=1}^{K} \prod_{j=1}^{i} a_{j}\right)\left(\sum_{i=K+2}^{N} \prod_{j=1}^{i} b_{j}\right) \geq\left(\sum_{i=K+2}^{N} \prod_{j=1}^{i} a_{j}\right)\left(\sum_{i=1}^{K} \prod_{j=1}^{i} b_{j}\right),
$$

and

$$
\sum_{i_{1}=1}^{K} \sum_{i_{2}=K+1}^{N}\left(\prod_{j=1}^{i_{1}} a_{j} \prod_{j=1}^{i_{2}} b_{j}\right) \geq \sum_{i_{1}=1}^{K} \sum_{i_{2}=K+1}^{N}\left(\prod_{j=1}^{i_{1}} b_{j} \prod_{j=1}^{i_{2}} a_{j}\right) .
$$

Notice that if $i_{1} \in\{1, \ldots, K\}$ and $i_{2} \in\{K+1, \ldots, N\}$, then

$$
\left(\prod_{j=1}^{i_{1}} a_{j} \prod_{j=1}^{i_{2}} b_{j}\right) \geq\left(\prod_{j=1}^{i_{1}} b_{j} \prod_{j=1}^{i_{2}} a_{j}\right)
$$

and hence the previous inequality holds.

Proof of Proposition 5. First we show the existence and uniqueness of a symmetric equilibrium. Let $\bar{u}(r)$ denote the probability of making the correct final decision, conditional on a voter being asked and reporting a signal corresponding to the true state of the world and the rest of the voters acquiring information with probability $r$ and reporting it truthfully upon being asked. Let $\underline{u}(r)$ denote the probability of making the correct final decision, conditional on a voter being asked and reporting a signal not corresponding to the true state of the world and the rest of the voters acquiring information with probability $r$ and reporting it truthfully upon being asked. The incentive compatibility constraint can be written as

$$
p \bar{u}(r)+(1-p) \underline{u}(r)-c \geq \frac{1}{2} \bar{u}(r)-\frac{1}{2} \underline{u}(r) .
$$

(This is because, if a voter acquires information, the probability that she gets the correct signal is $p$; otherwise, it is $1 / 2$.) The previous inequality can be rewritten as

$$
\left(p-\frac{1}{2}\right)(\bar{u}(r)-\underline{u}(r)) \geq c .
$$

If the previous inequality is strict for all $r \in[0,1]$, then when all voters acquire information with probability one is a symmetric equilibrium. Similarly, if the previous inequality never holds, when no voter acquires information is a symmetric equilibrium. Otherwise, in a symmetric mixed strategy equilibrium, the previous inequality must hold with equality. To prove the proposition, it is enough to show that $\bar{u}(r)-\underline{u}(r)$ is decreasing in $r$. (This is because from the Theorem of Intermediate Values the uniqueness of $r$ follows.)

$\bar{u}(r)-\underline{u}(r)$ is the difference between the utility of a voter who reported the right signal and the utility if she reported the wrong signal. Also notice that $\bar{u}(r)-\underline{u}(r)$ is simply the probability that a certain voter is decisive conditional on being asked. This is because, since the mechanism is defined by a decreasing function, whenever a voter is pivotal the final decision is right if and only if the voter reports the correct signal. In what follows we enumerate those sequences along which 
a certain voter is decisive and compute the probabilities of those sequences conditional on Voter 1 is being asked.

The precision of a report given that a voter acquires information with probability $r$ is

$$
\widehat{p}=r p+(1-r) \frac{1}{2}
$$

Let $\widehat{q}=1-\widehat{p}$.

Let $f$ be the function defining the mechanism. Consider a sequence $s=\left(s_{1}, s_{2}\right)$ such that $f\left(l\left(s_{1}\right)\right)-1=d\left(s_{1}\right)-1$. That is, if after $s_{1}$ a signal $\mathcal{H}$ is reported, action $a_{H}$ is taken. Furthermore, assume that $f(l(s))-1=|d(s)|-1$, and $d(s) \leq 0$. This means that if after the sequence $s_{1}$ a signal $\mathcal{L}$ is reported, action $a_{L}$ is taken. Notice the following: If nature specifies the signal sequence $s$ for the rest of the voters, Voter 1 is pivotal if her position is among the first $l\left(s_{1}\right)+1$ voters. The probability of such a sequence conditional on being asked and being among the first $l\left(s_{1}\right)+1$ voters is clearly

$$
\frac{l\left(s_{1}\right)+1}{\bar{L}}(\widehat{p} \widehat{q})^{\frac{l(s)-d(s)}{2}}\left(\widehat{p}^{f(l(s))-1}+\widehat{q}^{f(l(s))-1}\right) .
$$

Let $n\left(l_{1}, l\right)$ the number of those sequences $s=\left(s_{1}, s_{2}\right)$ for which $f\left(l\left(s_{1}\right)\right)-1=d\left(s_{1}\right)-1$ and $f(l(s))-1=-d(s)-1$, where $l$ denotes $l(s)$ and $l_{1}$ denotes $l\left(s_{1}\right)+1$. Then, $\bar{u}(r)-\underline{u}(r)$ can be written as

$$
\begin{aligned}
& \sum_{\left(l_{1}, l\right) \in \mathbb{N}^{2}} n\left(l_{1}, l\right) \frac{l_{1}}{\bar{L}}(\widehat{p} \widehat{q})^{\frac{l-f(l)}{2}}\left(\widehat{p}^{f(l)-1}+\widehat{q}^{f(l)-1}\right) \\
= & \sum_{\left(l_{1}, l\right) \in \mathbb{N}^{2}} \frac{l(\widehat{p} \widehat{q})^{\frac{l-f(l)}{2}}\left(\widehat{p}^{f(l)}+\widehat{q}^{f(l)}\right)}{\bar{L}}\left[\frac{l_{1}}{l} n\left(l_{1}, l\right) \frac{\widehat{p}^{f(l)-1}+\widehat{q}^{f(l)-1}}{\widehat{p}^{f(l)}+\widehat{q}^{f(l)}}\right] \\
= & \sum_{l \in \mathbb{N}} \frac{l(\widehat{p} \widehat{q})^{\frac{l-f(l)}{2}}\left(\widehat{p}^{f(l)}+\widehat{q}^{f(l)}\right)}{\bar{L}}\left[\sum_{l_{1} \in \mathbb{N}} \frac{l_{1}}{l} n\left(l_{1}, l\right) \frac{\widehat{p}^{f(l)-1}+\widehat{q}^{f(l)-1}}{\widehat{p}^{f(l)}+\widehat{q}^{f(l)}}\right] .
\end{aligned}
$$

Let us introduce the following two pieces of notations:

$$
\begin{aligned}
& a(l, \widehat{p})=\frac{l(\widehat{p} \widehat{q})^{\frac{l-f(l)}{2}}\left(\widehat{p}^{f(l)}+\widehat{q}^{f(l)}\right)}{\bar{L}} \\
& b(l, \widehat{p})=\sum_{l_{1} \in \mathbb{N}} \frac{l_{1}}{l} n\left(l_{1}, l\right) \frac{\widehat{p}^{f(l)-1}+\widehat{q}^{f(l)-1}}{\widehat{p}^{f(l)}+\widehat{q}^{f(l)}} .
\end{aligned}
$$

Hence we can rewrite $\bar{u}(r)-\underline{u}(r)$ as

$$
\sum_{l \in \mathbb{N}} a(l, \widehat{p}) b(l, \widehat{p}) .
$$

Next, we show that the hypothesis of the Lemma 11 is satisfied for $a$ and $b$. Notice that

$$
\begin{aligned}
\frac{d}{d \widehat{p}} \frac{\widehat{p}^{d-1}+\widehat{q}^{d-1}}{\widehat{p}^{d}+\widehat{q}^{d}} & =\frac{(d-1)\left(p^{2 d-2}-q^{2 d-2}+p^{d-2} q^{d}-p^{d} q^{d-2}\right)}{\left(\widehat{p}^{d-1}+\widehat{q}^{d-1}\right)^{2}} \\
-\frac{d\left(p^{2 d-2}-q^{2 d-2}\right)}{\left(\widehat{p}^{d-1}+\widehat{q}^{d-1}\right)^{2}} & <0 .
\end{aligned}
$$


Furthermore, $b(l, \widehat{p})$ is clearly increasing in $l$. It remains to show, that if $p>p^{\prime}$ then $a(., p)$ first-order stochastically dominates $a\left(., p^{\prime}\right)$. Observe that

$$
\begin{aligned}
\frac{a(l, p)}{a(l+1, p)} & =\frac{l(\widehat{p} \widehat{q})^{\frac{l-f(l)}{2}}\left(\widehat{p}^{f(l)}+\widehat{q}^{f(l)}\right)}{(l+1)(\widehat{p} \widehat{q})^{\frac{l+1-f(l+1)}{2}}\left(\widehat{p}^{f(l+1)}+\widehat{q}^{f(l+1)}\right)} \\
& =\frac{l}{l+1}(\widehat{p} \widehat{q})^{\frac{l-f(l)}{2}-\frac{l+1-f(l+1)}{2}} \frac{\widehat{p}^{f(l)}+\widehat{q}^{f(l)}}{\widehat{p}^{f(l+1)}+\widehat{q}^{f(l+1)}} .
\end{aligned}
$$

Recall that $f(l+1)=f(l)$ or $f(l)-1$. If $f(l+1)=f(l)$ then

$$
\frac{a(l, p)}{a(l+1, p)}=\frac{l}{l+1}(\widehat{p} \widehat{q})^{-\frac{1}{2}}
$$

otherwise

$$
\begin{gathered}
\frac{a(l, p)}{a(l+1, p)}=\frac{l}{l+1} \frac{\widehat{p}^{f(l)}+\widehat{q}^{f(l)}}{\widehat{p}^{f(l)-1}+\widehat{q}^{f(l)-1}} . \\
\frac{d}{d p} \frac{l}{l+1}(\widehat{p} \widehat{q})^{-\frac{1}{2}}>0, \\
\frac{d}{d p} \frac{l}{l+1} \frac{\widehat{p}^{f(l)}+\widehat{q}^{f(l)}}{\widehat{p}^{f(l)-1}+\widehat{q}^{f(l)-1}}>0 .
\end{gathered}
$$

Therefore by Lemma $12 a(., p)$ indeed first-order stochastically dominates $a\left(., p^{\prime}\right)$. Hence, Lemma 11 can be applied and one can conclude that $\bar{u}(r)-\underline{u}(r)$ is indeed decreasing in $r$.

The second part of the statement of the proposition follows from the continuity of (30) in $r$ and the continuity of (9) and (10) in the probabilities described in (8).

Proof of Lemma 9. We show that $e\left(M_{2}(K, 1)\right)>e\left(M_{1}(K, 1)\right)$ if $c=0$. Then the statement of the lemma follows from the continuity of the efficiency of any continuation mechanism in $c$.

Assume that $c=0$. First, we compute $e\left(M_{1}(K, 1)\right)$. Start with $M_{1}$. The change in the objective function is

$$
\begin{aligned}
\operatorname{dObj}\left(M_{1}(K, 1)\right) & =N[p(1) B(2)+q(1) B(1)-B(1)] \\
& =N p(1)[B(2)-B(1)]=N p q(p-q),
\end{aligned}
$$

where the last equality follows from Lemma 1 . The change in the incentive compatibility constraint can be written as

$$
\begin{aligned}
d I C\left(M_{1}(K, 1)\right)= & \frac{p-q}{4 p q}\{p(1)[B(2) 2-(p-q)(K+1)]+q(1) B(1)[1-(p-q)(K+2)] \\
& -B(1)[1-(p-q) K]\} .
\end{aligned}
$$

Again by Lemma 1, this can be rewritten as 


$$
\begin{aligned}
& \frac{p-q}{4 p q}\left[p^{2}(2-(p-q)(K+1))+2 p^{2} q(1-(p-q)(K+2))-p(1-(p-q) K]\right. \\
= & \frac{p-q}{4 p q}\left[2 p^{2}-p^{2}(p-q)(K+1)+2 p^{2} q-2 p^{2} q(p-q)(K+2)-p+p(p-q) K\right] \\
= & \frac{p-q}{4 p q}\left[(p-q)(K+2)\left(p-p^{2}-2 p^{2} q\right)+p^{2}(p-q)-2 p(p-q)+2 p^{2}+2 p^{2} q-p\right] \\
= & \frac{p-q}{4 p q}\left[(p-q)(K+2) p(q-2 p q)+(p-q) p(p-2)+p(2 p-1)+2 p^{2} q\right] \\
= & \frac{p-q}{4 p q}\left[-(p-q)^{2} p q(K+2)+(p-q) p(p-1)+2 p^{2} q\right] \\
= & \frac{p-q}{4 p q}\left[-(p-q)^{2} p q(K+2)+p q(2 p-(p-q))\right]=\frac{p-q}{4 p q}\left[-(p-q)^{2} p q(K+2)+p q\right]
\end{aligned}
$$

Therefore,

$$
\begin{aligned}
e\left(M_{1}(K, 1)\right) & =\frac{N p q(p-q)}{\frac{p-q}{4 p q}\left[(p-q)^{2} p q(K+2)-p q\right]} \\
& =\frac{4 N p q /(p-q)}{(p-q)(K+2)-\frac{1}{p q}} .
\end{aligned}
$$

Now we compute the efficiency of $M_{2}$. The change in the objective function is $\operatorname{dObj}\left(M_{2}(K, 1)\right)=N[-B(1)+P(\mathcal{H} \mid d=1) B(2)+(P(\mathcal{L}, \mathcal{H}, \mathcal{H} \mid d=1)+P(\mathcal{L}, \mathcal{L}, \mathcal{L} \mid d=1)) B(2)+$ $+(P(\mathcal{L}, \mathcal{H}, \mathcal{L}, \mathcal{H} \mid d=1)+P(\mathcal{L}, \mathcal{L}, \mathcal{H}, \mathcal{H} \mid d=1)+P(\mathcal{L}, \mathcal{L}, \mathcal{H}, \mathcal{L} \mid d=1)+P(\mathcal{L}, \mathcal{H}, \mathcal{L}, \mathcal{L} \mid d=1)) R(1)]$ where $P(s \mid d=1)$ is the probability of sequence $s$ conditional on $d=1$. The conditional probabilities appearing in the previous expression are:

$$
\begin{aligned}
P(\mathcal{H} \mid d & =1)=p(1)=p^{2}+q^{2} \\
P(\mathcal{L}, \mathcal{H}, \mathcal{H} \mid d & =1)=P(\mathcal{L} \mid d=1) P(\mathcal{H} \mid d=0) P(\mathcal{H} \mid d=1)=q(1) p(0) p(1)=p q\left(p^{2}+q^{2}\right) \\
P(\mathcal{L}, \mathcal{L}, \mathcal{L} \mid d & =1)=P(\mathcal{L} \mid d=1) P(\mathcal{L} \mid d=0) P(\mathcal{L} \mid d=-1)=q(1) q(0) q(-1)=p q\left(p^{2}+q^{2}\right) \\
P(\mathcal{L}, \mathcal{H}, \mathcal{L}, \mathcal{H} \mid d & =1)=P(\mathcal{L} \mid d=1) P(\mathcal{H} \mid d=0) P(\mathcal{L} \mid d=1) P(\mathcal{H} \mid d=0)= \\
& =q(1) p(0) q(1) p(0)=p^{2} q^{2} \\
P(\mathcal{L}, \mathcal{L}, \mathcal{H}, \mathcal{H} \mid d & =1)=P(\mathcal{L} \mid d=1) P(\mathcal{L} \mid d=0) P(\mathcal{H} \mid d=-1) P(\mathcal{H} \mid d=0)= \\
& =q(1) q(0) p(-1) p(0)=p^{2} q^{2} \\
P(\mathcal{L}, \mathcal{L}, \mathcal{H}, \mathcal{L} \mid d & =1)=P(\mathcal{L} \mid d=1) P(\mathcal{L} \mid d=0) P(\mathcal{H} \mid d=-1) P(\mathcal{L} \mid d=0)= \\
P(\mathcal{L}, \mathcal{H}, \mathcal{L}, \mathcal{L} \mid d & =1)=P(\mathcal{L} \mid d=1) P(\mathcal{H} \mid d=0) P(\mathcal{L} \mid d=1) P(\mathcal{L} \mid d=0)= \\
& =q(1) p(0) q(1) q(0)=p^{2} q^{2}
\end{aligned}
$$


Using (32),

$$
\begin{gathered}
\operatorname{dObj}\left(M_{2}(K, 1)\right)=N\left[-p+p^{2}+2 p^{3} q+4 p^{2} q^{3}\right]=N p\left[-q+2 p^{2} q+4 p q^{3}\right] \\
=N p q\left[-1+2 p^{2}+4 p q^{2}\right]=N p q\left[-p^{2}-q^{2}-2 p q+2 p^{2}+4 p q^{2}\right]= \\
=N p q\left[p^{2}-q^{2}-2 p q+4 p q^{2}\right]=N p q[p-q-2 p q(1-2 q)] \\
=N p q(p-q)(1-2 p q) .
\end{gathered}
$$

The change in the incentive compatibility constraint due to $M_{2}$ at $V(K, 1)$ is:

$$
\begin{aligned}
d I C\left(M_{2}(K, 1)\right)= & \frac{p-q}{4 p q}[-B(1)(1-(p-q) K)+P(\mathcal{H} \mid d=1) B(2)(2-(p-q)(K+1)) \\
& +(P(\mathcal{L}, \mathcal{H}, \mathcal{H} \mid d=1)+P(\mathcal{L}, \mathcal{L}, \mathcal{L} \mid d=1)) B(2)(2-(p-q)(K+3)) \\
+(P(\mathcal{L}, \mathcal{H}, \mathcal{L}, \mathcal{H} \mid d= & 1)+P(\mathcal{L}, \mathcal{L}, \mathcal{H}, \mathcal{H} \mid d=1)+P(\mathcal{L}, \mathcal{L}, \mathcal{H}, \mathcal{L} \mid d=1) \\
+P(\mathcal{L}, \mathcal{H}, \mathcal{L}, \mathcal{L} \mid d= & 1)) R(1)(-1-(p-q)(K+4))] .
\end{aligned}
$$

Using (32) again,

$$
\begin{aligned}
d I C\left(M_{2}(K, 1)\right)= & \frac{p-q}{4 p q}\left[-p(1-(p-q) K)+p^{2}(2-(p-q)(K+1))\right. \\
& +2 p^{3} q(2-(p-q)(K+3))+4 p^{2} q^{3}(-1-(p-q)(K+4)] \\
= & \frac{p-q}{4 p q}\left[-p+p(p-q) K+2 p^{2}-p^{2}(p-q)(K+1)\right. \\
& \left.+4 p^{3} q-2 p^{3} q(p-q)(K+3)-4 p^{2} q^{3}-4 p^{2} q^{3}(p-q)(K+4)\right] \\
= & \frac{p-q}{4 p q}\left[(p-q)(K+2)\left(p-p^{2}-2 p^{3} q-4 p^{2} q^{3}\right)\right. \\
& -2 p(p-q)+p^{2}(p-q)-2 p^{3} q(p-q) \\
& \left.-8 p^{2} q^{3}(p-q)-p+2 p^{2}+4 p^{3} q-4 p^{2} q^{3}\right] \\
= & \frac{p-q}{4 p q}\left[(p-q)(K+2) p\left(q-2 p^{2} q-4 p q^{3}\right)\right. \\
& \left.-(p-q)\left(2 p-p^{2}+2 p^{3} q+8 p^{2} q^{3}\right)-p+2 p^{2}+4 p^{3} q-4 p^{2} q^{3}\right] \\
= & \frac{p-q}{4 p q}\left[(p-q)(K+2) p q\left(1-2 p^{2}-4 p q^{2}\right)\right. \\
& \left.-(p-q) p\left(2-p+2 p^{2} q+8 p q^{3}\right)+p\left(2 p+4 p^{2} q-4 p q^{3}-1\right)\right] \\
= & \frac{p-q}{4 p q}[(p-q)(K+2) p q(-(p-q)(1-2 p q)) \\
& \left.-(p-q) p\left(1+q+2 p^{2} q+8 p q^{3}\right)+p\left(2 p+4 p^{2} q-4 p q^{3}-1\right)\right] \\
= & \frac{p-q}{4 p q}\left[-(p-q)^{2} p q(K+2)(1-2 p q)\right. \\
& \left.-(p-q) p\left(1+q+2 p^{2} q+8 p q^{3}\right)+p\left(2 p+4 p^{2} q-4 p q^{3}-1\right)\right] .
\end{aligned}
$$


Hence,

$$
\begin{gathered}
e\left(M_{2}(K, 1)\right) \\
=\frac{4 N(p q)^{2}(1-2 p q)}{\left[(p-q)^{2} p q(K+2)(1-2 p q)+(p-q) p\left(1+q+2 p^{2} q+8 p q^{3}\right)-p\left(2 p+4 p^{2} q-4 p q^{3}-1\right)\right]} \\
=\frac{4 N p q /(p-q)}{\left[(p-q)(K+2)-\frac{p\left(2 p+4 p^{2} q-4 p q^{3}-1\right)-(p-q) p\left(1+q+2 p^{2} q+8 p q^{3}\right)}{p q(p-q)(1-2 p q)}\right]} .
\end{gathered}
$$

From (31) and (33), to prove that $e\left(M_{1}(K, 1)\right)<e\left(M_{2}(K, 1)\right)$, we have to show that

$$
\frac{1}{(p-q)}-\frac{p\left(2 p+4 p^{2} q-4 p q^{3}-1\right)-(p-q) p\left(1+q+2 p^{2} q+8 p q^{3}\right)}{p q(p-q)(1-2 p q)}<0 .
$$

Notice that

$$
\begin{aligned}
& \frac{1}{(p-q)}-\frac{p\left(2 p+4 p^{2} q-4 p q^{3}-1\right)-(p-q) p\left(1+q+2 p^{2} q+8 p q^{3}\right)}{p q(p-q)(1-2 p q)} \\
= & \frac{p q(1-2 p q)-p\left(2 p+4 p^{2} q-4 p q^{3}-1\right)+(p-q) p\left(1+q+2 p^{2} q+8 p q^{3}\right)}{p q(p-q)(1-2 p q)} \\
= & \frac{q-2 p q^{2}-2 p-4 p^{2} q+4 p q^{3}+p+q+p-q+p q-q^{2}+2 p^{3} q-2 p^{2} q^{2}+8 p q^{3}(p-q)}{q(p-q)(1-2 p q)} \\
= & \frac{q-2 p q^{2}-4 p^{2} q+4 p q^{3}+p q-q^{2}+2 p^{3} q-2 p^{2} q^{2}+8 p q^{3}(p-q)}{q(p-q)(1-2 p q)} \\
= & \frac{2 p q-2 p q^{2}-4 p^{2} q+4 p q^{3}+2 p^{3} q-2 p^{2} q^{2}+8 p q^{3}(p-q)}{q(p-q)(1-2 p q)} \\
= & \frac{-2 p^{2} q+4 p q^{3}+2 p^{3} q-2 p^{2} q^{2}+8 p q^{3}(p-q)}{q(p-q)(1-2 p q)}=\frac{2 p q\left(2 q^{2}+p^{2}-p q-p\right)+8 p q^{3}(p-q)}{q(p-q)(1-2 p q)} \\
= & \frac{2 p q\left(2 q^{2}-2 p q\right)+8 p q^{3}(p-q)}{q(p-q)(1-2 p q)}=\frac{(p-q)\left(-4 p q^{2}+8 p q^{3}\right)}{q(p-q)(1-2 p q)}=-\frac{4 p q^{2}(p-q)^{2}}{q(p-q)(1-2 p q)} \\
= & -\frac{4 p q(p-q)}{(1-2 p q)}<0,
\end{aligned}
$$

where the inequality follows from $p q \leq \frac{1}{4}$.

Proof of Lemma 10. First, we claim that for all $c>0$, there exists an $N \in \mathbb{N}$ such that if the cost of information acquisition is $c$ and there are more than $N$ voters, the maximum number of voters asked in the ex-post optimal mechanism is strictly smaller than $N / 2$. This follows from exactly the same argument as the one used in the proof of Proposition 3 to show that the maximum number of voters asked is finite.

Let us fix $\bar{c}>0$ and $N \in \mathbb{N}$ such that there is an optimal ex-post efficient mechanism $M^{*}(\bar{c})$ where the maximum number of voters asked is $K<N / 2$. Furthermore, for each $c(>0)$, let $M^{*}(c)$ denote an ex-post efficient mechanism if the cost of information acquisition is $c$ and the number of voters is $N$. Let $Z(M)$ denote the maximum number of voters asked in the mechanism $M$. Let us define $c^{*}$ as

$$
c^{*}=\sup \left\{c \mid Z\left(M^{*}(c)\right)=K\right\} .
$$


Notice that $c^{*}$ is well-defined since $Z\left(M^{*}(\bar{c})\right)=K$.

Choose a sequence $\left\{c_{n}\right\}_{1}^{\infty}, c_{n}>c^{*}$, that converges to $c^{*}$. Furthermore, $M^{*}\left(c_{n}\right)$ involves randomization for all $n \in \mathbb{N}$. (This is possible because of Lemma 8.) Let $\left\{c_{n_{k}}\right\}_{1}^{\infty}$ be a subsequence of $\left\{c_{n}\right\}_{1}^{\infty}$ such that $M\left(c_{n_{k}}\right)$ converges. This is possible by Proposition 4 . Let the limit mechanism be denoted by $\widetilde{M}$, which is optimal if the cost of information acquisition is $c^{*}$, again by Proposition 4. By the definition of $c^{*}, Z\left(M^{*}\left(c_{n}\right)\right)<K$, and therefore $Z(\widetilde{M})<K$.

We show that $M_{1}$ is used at $V(K-2,1)$ with probability strictly between zero and one either in an optimal mechanism if the cost is $c^{*}$, or if the cost is slightly below $c^{*}$.

By Proposition 4 and the definition of $c^{*}$, there exists a sequence $\left\{c_{n}^{\prime}\right\}_{1}^{\infty}$ such that

$$
\left\{c_{n}^{\prime}\right\}_{1}^{\infty} \subset\left\{c \mid Z\left(M^{*}(c)\right)=K\right\},
$$

$c_{n}^{\prime} \leq c^{*},\left\{c_{n}^{\prime}\right\}_{1}^{\infty}$ is converging to $c^{*}$, and $M^{*}\left(c_{n}^{\prime}\right)$ is converging to an optimal mechanism $\bar{M}$ if the cost is $c^{*}$. (If $c^{*}$ is an isolated point in the set $\left\{c \mid Z\left(M^{*}(c)\right)=K\right\}$, then $c_{n}^{\prime}$ can be chosen to be identically $c^{*}$.) If $M_{1}$ is employed at $V(K-2,1)$ with probability strictly between zero and one in some $M^{*}\left(c_{n}^{\prime}\right)$, we are done. Suppose that $M_{1}$ at $V(K-2,1)$ is employed with probability one in $M^{*}\left(c_{n}^{\prime}\right)$ for all $n \in \mathbb{N}$. Then $M_{1}$ is employed at $V(K-2,1)$ with probability one in $\bar{M}$.

However, the probability mixture of $\bar{M}$ and $\widetilde{M}$ is also an optimal mechanism for $c^{*}$. That is, it is also optimal for the SP to play $\bar{M}$ and $\widetilde{M}$ each with probability one-half. In that mechanism, however, there must exist a continuation mechanism that is employed with probability strictly between zero and one and that reaches $V(K, 1)$. From Theorem 1, however, it follows that this continuation mechanism must be $M_{1}$ at $V(K-2,1)$.

\section{Appendix B: The Proof of Theorem 1}

Let $\bar{d}$ denote the largest $d$ for which there exists an $l$ such that $p\left(V(l, d), M^{*}\right)>0$. For each $d,(d \in\{0, \ldots, \bar{d}-1\})$, let $\alpha(d)$ denote the largest possible $l$ for which $\rho\left(V(l, d), C, M^{*}\right)$ (and by symmetry $\left.\rho\left(V(l,-d), C, M^{*}\right)\right)$ is positive. Since there exists a state $V(l, \bar{d})$ such that $p\left(V(l, \bar{d}), M^{*}\right)>0$ for all $d(|d| \in\{0, \ldots, \bar{d}-1\})$, there must exist an $l$ such that $\rho\left(V(l, d), C, M^{*}\right)>$ 0 . (Otherwise the state $V(l, \bar{d})$ could not have been reached with positive probability.) Hence the function $\alpha$ is well defined. Next, we show that conditional on reaching $V(l, d)$, the mechanism continues at $V(l, d)$ if and only if $l<\alpha(d)$.

Lemma 13 Suppose $p\left(V(l, d), M^{*}\right)>0$. If $\alpha(|d|) \geq l$, then $p\left(V(l, d), C, M^{*}\right)>0$. If $\alpha(|d|)<l$, then $p\left(V(l, d), C, M^{*}\right)=0$.

Proof. Without loss of generality, assume $d \geq 0$. First, suppose that $p\left(V(l, d), M^{*}\right)>0$ and $\alpha(d)>l$. Assume, by contradiction, that $p\left(V(l, d), C, M^{*}\right) \neq 1$. (That is, $p\left(V(l, d), M a j, M^{*}\right)>$ 0 .) By the definition of the function $\alpha$, a continuation mechanism is employed at the state 
$V(\alpha(d), d)$ with positive probability. Since $p\left(V(l, d), M^{*}\right)>0$ and $\alpha(d)<l$, this continuation mechanism is also feasible at the state $V(l, d)$. Furthermore, by Lemma 5 , the continuation mechanism is more efficient at $V(l, d)$ than at $V(\alpha(d), d)$. But then, by Lemma 6 , $p\left(V(l, d), M a j, M^{*}\right)>0$ is impossible. If $\alpha(d)=l$, then by the definition of the function $\alpha$, $p\left(V(l, d), C, M^{*}\right)>0$.

If $\alpha(d)<l$, then the mechanism stops at $V(l, d)$ by the definition of $\alpha$.

Lemma 14 The function $\alpha$ is strictly decreasing.

Proof. Suppose, by contradiction, that $d>d^{\prime}(\geq 0)$ and $\alpha(d) \geq \alpha\left(d^{\prime}\right)$. Notice that the mechanism continues at the state $V(\alpha(d), d)$. However, after continuing from $V(\alpha(d), d)$, whenever the difference between the numbers of different signals is $d^{\prime}$, the mechanism must stop. (This is because $\alpha\left(d^{\prime}\right)<\alpha(d)$.) Hence the decision of the SP after reaching $V(\alpha(d), d)$ is always the majority decision at $V(\alpha(d), d)$, no matter what the voters report in the continuation mechanism. This contradicts the statement of Lemma 7.

Lemma 13 only characterized those states where the mechanism continues conditional on reaching those states in terms of the function $\alpha$. The next lemma essentially says that the states characterized in Lemma 13 are actually reached.

Lemma 15 Let $V(l, d) \in \mathcal{V}(N)$. Then

$$
\rho\left(V(l, d), C, M^{*}\right)>0 \Leftrightarrow l \leq \alpha(|d|) .
$$

Proof. If $\rho\left(V(l, d), C, M^{*}\right)>0$, then by the definition of the function $\alpha, l \leq \alpha(|d|)$.

Suppose that there exists a state $V(l, d) \in \mathcal{V}(N)$ such that $l \leq \alpha(|d|)$ but $\rho\left(V(l, d), C, M^{*}\right)=$ 0 . That is, the set

$$
A=\left\{V(l, d): V(l, d) \in \mathcal{V}(N), l \leq \alpha(|d|), \rho\left(V(l, d), C, M^{*}\right)=0\right\}
$$

is non-empty. Among such states, consider the set $\widetilde{A}$ of those where $l+|d|$ is minimal. That is,

$$
\widetilde{A}=\arg \min _{V \in A}\{l(V)+|d(V)|\} .
$$

Among these states, consider one where $l$ is minimal. Let $V\left(l^{\prime}, d^{\prime}\right)$ be such a state. That is,

$$
V\left(l^{\prime}, d^{\prime}\right) \in \arg \min _{V \in \widetilde{A}}\{l(V)\} .
$$

Since the mechanism is symmetric with respect to $\mathcal{H}$ and $\mathcal{L}, d^{\prime} \geq 0$ can be assumed. There can be two reasons why $\rho\left(V\left(l^{\prime}, d^{\prime}\right), C, M^{*}\right)=0$ : either the state $V\left(l^{\prime}, d^{\prime}\right)$ is never reached, or although it is reached, the mechanism stops there. First, suppose that $p\left(V\left(l^{\prime}, d^{\prime}\right), M^{*}\right)>0$. Then, by Lemma 13, $\rho\left(V\left(l^{\prime}, d^{\prime}\right), C, M^{*}\right)>0$, a contradiction. Hence, $p\left(V\left(l^{\prime}, d^{\prime}\right), M^{*}\right)=0$. Then $\rho\left(V\left(l^{\prime}-1, d^{\prime}-1\right), C, M^{*}\right)=0$, for otherwise the state $V\left(l^{\prime}, d^{\prime}\right)$ would be reached with positive probability. We consider two different cases. 
Case 1: $d^{\prime}>0$. Then, since $l^{\prime} \leq \alpha\left(d^{\prime}\right)$, from Lemma 14 it follows that $l^{\prime}-1 \leq \alpha\left(d^{\prime}-1\right)$. That is, $V\left(l^{\prime}-1, d^{\prime}-1\right) \in A$. Since $l^{\prime}-1+d^{\prime}-1<l^{\prime}+d^{\prime}, V\left(l^{\prime}, d^{\prime}\right) \notin \widetilde{A}$, a contradiction.

Case 2: $d^{\prime}=0$. Then $p\left(V\left(l^{\prime}-1,-1\right), M^{*}\right)$ must be zero. (If not, then since $V\left(l^{\prime}, 0\right) \in \widetilde{A}$, $V\left(l^{\prime}-1,-1\right) \in \widetilde{A}$ also. But then

$$
V\left(l^{\prime}, 0\right) \notin \arg \min _{V \in \widetilde{A}}\{l(V)\},
$$

a contradiction.) Since $p\left(V\left(l^{\prime}-1,-1\right), M^{*}\right)=0$, it follows that the mechanism cannot continue at $V\left(l^{\prime}-2,0\right)$. That is, $\rho\left(V\left(l^{\prime}-2,0\right), C, M^{*}\right)=0$. This implies $V\left(l^{\prime}, 0\right) \notin \widetilde{A}$, again a contradiction.

Let $\bar{W}$ denote the set of states where the mechanism involves randomization:

$$
\bar{W}=\left\{V: \rho\left(V, C, M^{*}\right), \rho\left(V, M a j, M^{*}\right)>0\right\} .
$$

Lemma 16 Let $V(\widehat{l}, \widehat{d}) \in \bar{W}$. Then $\alpha(|\widehat{d}|)=\widehat{l}$.

Proof. Since $\rho\left(V(\widehat{l}, \widehat{d}), C, M^{*}\right)>0, \widehat{l} \leq \alpha(|\widehat{d}|)$ by Lemma 15. Suppose that $\widehat{l}<\alpha(|\widehat{d}|)$. By the definition of the function $\alpha, \rho\left(V(\alpha(|\widehat{d}|), \widehat{d}), C, M^{*}\right)>0$. Notice that the continuation mechanism induced by $M^{*}$ at $V(\alpha(|\widehat{d}|), \widehat{d})$ is feasible at $V(\widehat{l}, \widehat{d})$ and more efficient by Lemma 5. Since $\rho\left(V(\widehat{l}, \widehat{d}), M a j, M^{*}\right)>0$, this contradicts Lemma 6 , and therefore $\alpha(|\widehat{d}|)=\widehat{l}$.

The function $f$ in Theorem 1 can be defined as follows:

$$
f(l)=\min \{d \mid \bar{d}>d \geq 1, \alpha(d) \leq l\} .
$$

We are ready to prove Theorem 1.

Proof of Theorem 1. We have to show that the function $f$ defined by (34) satisfies the claim of the theorem. Since $\alpha$ is decreasing (by Lemma 14) the function $f$ is also decreasing. Next, we show that

$$
f(l+1)=f(l) \text { or } f(l+1)=f(l)-1 .
$$

If $l+1=\alpha(d)$ then, by (34), $f(l+1)=d$. Since $\alpha(d)>l$ and $\alpha$ is decreasing, it follows from (34) that $f(l)>d$. But $\alpha(d+1)<\alpha(d)=l+1$. Therefore,

$$
f(l)=d+1=f(l+1)+1 .
$$

If $f(l+1)=d$ and $l+1<\alpha(d)$, then clearly $l \leq \alpha(d)$. Hence $f(l)=d=f(l+1)$. From this argument, it follows that the set $W$ in the claim of Theorem 1 can be defined as

$$
W=\{V: \alpha(|d(V)|)=l(V)\} .
$$

$f(N)$ is one for the following reason. From (34), it follows that $f(N) \geq 1$. Suppose, by contradiction, that $f(N)>1$. Then at a state $V(N-1, d)$ where $d \geq 1$, an additional voter is 
asked to collect information even though she cannot change the posterior of the SP. This contradicts to the statement of Lemma 7.

It remains to show that conditions (i)-(iii) are also satisfied.

(i) Suppose that $f(l) \leq d$ and $V(l, d) \notin W$. Also assume that $p\left(V(l, d), M^{*}\right)>0$. Suppose, by contradiction, that the mechanism continues with positive probability, that is, $p\left(V(l, d), C, M^{*}\right)>$ 0 . But then $\alpha(|d|) \geq l$ by the definition of $\alpha$. If $\alpha(|d|)=l$, then $V(l, d) \in W$ by (35), a contradiction. If $\alpha(d)>l$, then $f(l)=d$ is impossible by the definition of $f$.

(ii) Suppose that $f(l)<d, V(l, d) \notin W$, and $p\left(V(l, d), M^{*}\right)>0$. Since $f(l)>d$ and $V(l, d) \notin W$, it follows that $\alpha(d)>l$. From Lemma $15 p\left(V(l, d), C, M^{*}\right)>0$ follows. By Lemma 16, $p\left(V(l, d), M a j, M^{*}\right)=0$. Hence $p\left(V(l, d), C, M^{*}\right)=1$.

(iii) This follows immediately from (35) and Lemma 16.

From Lemma 8, it follows that there exists an optimal ex-post efficient mechanism that involves randomization at only a single state. Hence, for this mechanism, there is a single state $V \in W$ such that $p\left(V, M a j, M^{\star}\right)>0$.

\section{References}

[1] Austen-Smith, D., and J.S. Banks (1996), "Information Aggregation, Rationality, and the Condorcet Jury Theorem," American Political Science Review 90, 34-45.

[2] Austen-Smith, D., and T. Federsen (2002), "The Inferiority of Deliberation under Unanimity Rule," Mimeo, Northwestern University.

[3] Bergemann, D., and J. Valimaki (2002), "Information Acquisition and Efficient Mechanism Design," Econometrica, 70, 1007-1033.

[4] Cai, H. (2003), "Costly Participation and Heterogeneous Preferences in Informational Committees," Discussion Paper 257, UCLA Department of Economics.

[5] Chwe, M. (1999), "Minority Voting Rights Can Maximize Majority Welfare," American Political Science Review, 93(1), 85-97.

[6] Cremer, J., and R. McLean (1988), "Full Extraction of the Surplus in Bayesian and Dominant Strategy Auctions," Econometrica, 56, 1247-1258.

[7] DeGroot, M. H. (1970), Optimal Statistical Decisions, McGraw-Hill, New York.

[8] Dekel, E., and M. Piccione (2000), "Sequential Voting Procedures in Symmetric Binary Elections," Journal of Political Economy, 108(1), 34-55.

[9] Federsen, T. J., and W. Pesendorfer (1996), "The Swing Voter's Curse," American Economic Review, 86(3), 408-424. 
[10] Federsen, T. J., and W. Pesendorfer (1997), "Voting Behavior and Information Aggregation in Elections with Private Information," Econometrica, 65(5), 1029-1058.

[11] Federsen, T. J., and W. Pesendorfer (1998), "Convicting the Innocent: The Inferiority of Unanimous Jury Verdicts under Strategic Voting," American Political Science Review, 92, 23-35.

[12] Gerardi, D., and L. Yariv (2003), "Committee Design in the Presence of Communication," Cowles Foundation Discussion Paper No. 1411.

[13] Li, H. (2001), "A Theory of Conservatism," Journal of Political Economy, 109(3), 617-636.

[14] Li, H., S. Rosen and W. Suen (2001), "Conflicts and Common Interests in Committees," American Economic Review 91(5), 1478-1497.

[15] McAfee, R. P., and P. J. Reny (1992), "Correlated Information and Mechanism Design," Econometrica, 60(2), 395-421.

[16] McLennan, A. (1998), "Consequences of the Condorcet Jury Theorem for Beneficial Information Aggregation by Rational Agents," American Political Science Review 92(2), 413-418.

[17] Milgrom, P. (1981), "Rational Expectations, Information Acquisition, and Competitive Bidding," Econometrica 49, 921-943.

[18] Myerson, R. B. (1986), "Multistage Games with Communication," Econometrica 56(2), 323358.

[19] Mukhopadhaya, K. (2003), "Jury Size and the Free Rider Problem," The Journal of Law, Economics, \& Organization, 19(1), 24-44.

[20] Persico, N. (2000), "Information Acquisition in Auctions," Econometrica, 68(1), 135-148.

[21] Persico, N. (2004), "Committee Design with Endogenous Information," Review of Economic Studies, 71(1), 165-194.

[22] Smorodinsky, R., and M. Tennenholtz (2003), "Overcoming Free Riding in Multi-Party Computations-The Anonymous Case," Mimeo, Technion. 